\title{
Smoothed particle hydrodynamics (SPH) model for coupled analysis of a damaged ship with internal sloshing in beam seas
}

\author{
Cite as: Phys. Fluids 31, 032103 (2019); doi: 10.1063/1.5079315 \\ Submitted: 30 October 2018 - Accepted: 21 February 2019 • \\ Published Online: 12 March 2019
}

\author{
X. Y. Cao, ${ }^{1,2}$ (D) L. Tao, ${ }^{1,2, a)}$ (D) A.-M. Zhang, ${ }^{1}$ and F. R. Ming
}

\author{
AFFILIATIONS \\ ${ }^{1}$ College of Shipbuilding Engineering, Harbin Engineering University, Harbin 150001, China \\ ${ }^{2}$ Department of Naval Architecture, Ocean and Marine Engineering, University of Strathclyde, Glasgow G4 OLZ, United Kingdom
}

a) Author to whom correspondence should be addressed: longbin.tao@strath.ac.uk.

\begin{abstract}
The flooding of a damaged ship in waves is a complex process, often coupled with the internal and external liquid motion together with the ship hull motion. Paramount to the operation safety, in order to improve the prediction accuracy of ship motion during the flooding process, the strip theory is applied to study the dynamic response of the damaged ship in beam seas; a smoothed particle hydrodynamics (SPH) model is developed to consider the coupling effects of various factors including internal sloshing of intact cabins and damaged cabins and external waves. The numerical wave tank with a perfectly matched layer absorbing boundary condition is established and validated by the experimental results. The detailed sensitivity study is carried out focusing on the effects of damaged opening sizes, the relative position of opening, and the incident wave and the liquid loading conditions on the dynamic response of the damaged ship in regular beam waves. It is observed that the flooding process was slowed down and interrupted by the water exchanges at the damaged opening due to the dynamic motion. Compared with the opening facing the incident wave, the back one endangered the ship pronouncedly with large amplitude and frequency roll motion. It is also revealed that the liquid tank in the damaged ship imposes a significant influence on its rolling response. It is further demonstrated that the present SPH model is capable of handling the nonlinear phenomenon in a flooding process of a damaged ship.
\end{abstract}

Published under license by AIP Publishing. https://doi.org/10.1063/1.5079315

\section{INTRODUCTION}

When the ships are sailing in rough seas or river, the ship hull may be damaged due to collision, grounding, and other factors. $^{1-4}$ Such accidents can result in a large opening in the ship hull, and an abrupt flooding occurs. The dynamic response of a damaged ship in waves is a complex fluid-structure interaction problem, ${ }^{5-7}$ often influenced by the wave excitation and the internal liquid loads due to water flooding and sloshing. The flooding process in a damaged compartment consists of the inflow, inflooding motions, and progression ${ }^{8}$ may be accompanied with nonlinear flow behaviors, e.g., inflooding jet, sloshing, and free surface breaking. The dynamic motion of internal and external liquid loads and ship hull are strongly coupled, which can be a severe risk to the ship stability ${ }^{8}$ and even cause the ship to sink.

For the large opening cases, the abrupt flooding can be fast and affect the ship response. ${ }^{9}$ As demonstrated in the model tests on the damaged ship with large openings, ${ }^{9-11}$ the important role of the inflooding jet has been observed, which can determine the direction of the first roll in the transient stage and even for the final equilibrium position. The dynamic flow propagation inside the cabin can further increase the complexity of the flooding process. ${ }^{8,12}$ Previous studies have demonstrated that the hydrodynamic effects, including water inflow/outflow through the damage opening and floodwater accumulation, can influence the recovery moment of the ship hull. ${ }^{12,13}$ There are strong nonlinear effects on the response of the damaged ship at this stage due to the flooding dynamics. ${ }^{14-16}$ It is noted that many previous research studies were based on the assumption of flat horizontal free surface. However, Santos and Guedes Soares $^{17}$ stated that the calculated moment is much smaller than the dynamic roll moment, and it is necessary to take the dynamic fluid motion into account. Thus, further investigation is needed on the flooded water motions and progressions inside the damaged cabin. ${ }^{13}$ 
Compared to an intact ship in waves, a less severe roll motion for the damaged ship in waves was observed ${ }^{6,18,19}$ owing to the additional damping caused by flooding water. ${ }^{20}$ Different wave conditions can cause different patterns of flooding water motion, and the coupled responses of a damaged ship become more complex. ${ }^{15,21,22}$ Many factors intensify the behavior and affect the stability of a damaged ship in waves, e.g., the internal sloshing, the initial heeling angle during the damage creation, and the air flow. The coupling of liquid sloshing and ship motions has been widely studied;23,24 however, very limited studies have been reported on the damaged ship with liquid cabins in waves. It is noted that the existence of the air flow may restrain the fluid motion inside a damaged cabin ${ }^{25,26}$ by reducing the kinematic energy of inflow water. ${ }^{27}$ To focus on the coupling effect of the internal sloshing of the damaged cabin and the external fluid flow, the air flow and compression are not considered in this study assuming that the cabin is fully ventilated. Due to the complexity of the dynamic flooding process, the forward speed of the damaged ship is not taken into account. The coupling motion of roll-sway-heave on the ship is allowed under the external and internal fluid force. The dynamic flooding process coupled with ship motion in waves involves nonlinear characteristics such as sloshing, surface breaking, and splashing. It poses a great challenge for these factors being considered properly and implemented in a numerical method. ${ }^{28-30}$ The meshless smoothed particle hydrodynamics (SPH) method ${ }^{31-34}$ has advantages to deal with the nonlinear interaction with large free-surface deformation, ${ }^{35,36}$ violent impact, ${ }^{37,38}$ and other nonlinear phenomena. ${ }^{39,40}$ A wide validation of the SPH model against different sloshing flow conditions was conducted. ${ }^{35,41}$ The energy dissipated ${ }^{42,43}$ by the fluid in a coupled fluid-structure system and the energy balance ${ }^{44}$ for a floating body in waves were examined using this method. Some recent research studies on the damaged flooding process were conducted by using the SPH method. ${ }^{22,45-47}$ These studies showed the ability of the SPH method on this problem.

This paper presents an SPH model developed to study the coupling effect of flooding water, internal sloshing, and external waves on the dynamic response of a damaged ship. The paper is organized as follows: after the Introduction, Sec. II describes the SPH model development based on strip theory. In addition, a numerical wave tank with a Perfectly Matched Layer (PML) ${ }^{48,49}$ absorbing boundary condition is established. The prediction of wave load and floating body motion is validated by the experiment results and theoretical solution in Sec. III. In Sec. IV, effects of the parameters related to the damaged ship hull and incident wave properties including the opening sizes, the relative position of the opening, the incident wave conditions, and the liquid loading on the dynamic response of a damaged ship are examined.

\section{THEORETICAL BACKGROUND}

\section{A. SPH equation for the fluid}

A Lagrangian particle method SPH is introduced to solve both the internal and external flows interacting with the ship hull. The SPH model adopted ${ }^{50-52}$ is built based on the assumption of the fluid being barotropic, weakly compressible, and inviscid, with an artificial speed of sound selected to reduce compressible effects. The flow evolution is then governed by the continuity equation and the
Euler equation closed with a state equation which is the function of density,

$$
\begin{aligned}
& \frac{D \rho}{D t}=-\rho \nabla \cdot \boldsymbol{v}, \\
& \frac{D \boldsymbol{v}}{D t}=\boldsymbol{g}-\frac{1}{\rho} \nabla P, \\
& P=f(\rho),
\end{aligned}
$$

where $\rho$, $v$, and $P$ represent the density, the velocity, and the pressure, respectively; $\boldsymbol{g}$ is the body force per unit mass. In the SPH context, the Lagrangian discretized forms of the governing equations are adopted as follows: ${ }^{52}$

$$
\begin{gathered}
\frac{D \rho_{i}}{D t}=-\rho_{i} \sum_{j}\left(\boldsymbol{v}_{j}-\boldsymbol{v}_{i}\right) \cdot \nabla W_{i j} \frac{m_{j}}{\rho_{j}}, \\
\frac{D \boldsymbol{v}_{i}}{D t}=-\frac{1}{\rho_{i}} \sum_{j}\left(P_{i}+P_{j}\right) \nabla W_{i j} \frac{m_{j}}{\rho_{j}}+\boldsymbol{g}+\mathbf{F}_{v}, \\
P_{i}=\frac{c_{f}^{2} \rho_{0}}{\gamma}\left[\left(\frac{\rho_{i}}{\rho_{0}}\right)^{\gamma}-1\right],
\end{gathered}
$$

where $i, j$ represent a pair of interacting particles and $m$ and $W$ represent the mass and the weight kernel, respectively. In this study, $\boldsymbol{g}$ $=(0,-9.81) \mathrm{m} / \mathrm{s}^{2}$ represents the acceleration of gravity. To improve the computational stability, an artificial viscous $\operatorname{term}^{53} \boldsymbol{F}_{v}$ is added in the momentum equation,

$$
\mathbf{F}_{v}=\alpha h \sum_{j} m_{j} \frac{c_{i}+c_{j}}{\rho_{i}+\rho_{j}} \frac{\left(\boldsymbol{v}_{i}-\boldsymbol{v}_{j}\right) \cdot\left(\boldsymbol{r}_{i}-\boldsymbol{r}_{j}\right)}{\left|\boldsymbol{r}_{i}-\boldsymbol{r}_{j}\right|^{2}+(0.01 h)^{2}} \nabla W_{i j},
$$

where $\alpha$ is the artificial viscosity coefficient and $\alpha=0.02$ is chosen for all the simulations. $h$ is the smoothing length, $r$ is the vector radius, and $c$ is the speed of sound. The fluid is inviscid rotational flow. The Tait equation (4) ${ }^{54}$ is used by regarding the fluid as weakly compressible media, where $\gamma=7$ and $c_{f}$ is the reference speed of sound. In the weakly compressible SPH model, an artificial $c_{f}$ is generally chosen instead of the real one $e^{55}$ which fulfils the density variations less than 0.01 . $c_{f}$ can be estimated by the constraint of the static pressure and the kinetic energy pressure $^{56,57}$

$$
c_{f} \geq 10 \max \left(\sqrt{g H}, \max \left(\left\|\boldsymbol{v}_{f}\right\|\right)\right),
$$

where $\sqrt{g H}(H$ being the water depth) is constrained by the static pressure $\rho g H$ and $\max \left(\left\|\boldsymbol{v}_{f}\right\|\right)$ is the maximum flow velocity constrained by the kinetic energy $\rho v^{2} \cdot c_{f}=10 \sqrt{g H}$ is chosen in this study to satisfy this condition. More detailed derivation and choice of the governing equation can be seen in Refs. 58-60. For the selection of the kernel function, the Wendland kernel and the improved Gaussian kernel are good choice for the simulation of free surface flows. ${ }^{37,39,59,61}$ The improved Gaussian kernel is used aiming at lower computational cost, ${ }^{56}$ which is important for long duration simulations. For the cases studied in this paper, the flow in each segment (see Sec. II B) is two-dimensional, and the form $^{62}$ is

$$
W(r, h)=\frac{\left(e^{-(r / h)^{2}}-e^{-9}\right)}{\pi h^{2}\left(1-10 e^{-9}\right)}, \quad r \leq 3 h,
$$


where $r=\left|\boldsymbol{r}_{i}-\boldsymbol{r}_{j}\right|$ represents the distance between a pair of interacting particles and the smoothing length $h=1.23 d x$, where $d x$ is the initial particle spacing. The renormalized kernel equals to zero strictly at the cut-off radius $r=3 h$ of the compact support domain.

According to the above equations, the pressure of the flow field is determined by density; small changes in density can cause large fluctuations in the pressure field. Especially for the problem of a damaged ship in waves, spurious pressure noise will occur in the pressure field of the fluid particles after a long duration simulation. In order to remove the spurious numerical high-frequency oscillations, the improved moving least square (MLS) ${ }^{51}$ is used to reinitialize the density,

$$
\rho_{i}^{r e-i n}=\sum_{j} \rho_{j} W_{i j}^{M L S} m_{j} / \rho_{j}=\sum_{j} m_{j} W_{i j}^{M L S},
$$

where $W^{M L S}$ is the MLS correction kernel, ${ }^{51,63}$ and the correction is performed every 20 steps. It must be pointed that an artificial diffusive term ${ }^{35,37,64-66}$ added into the continuity equation is proposed instead of the density re-normalization, which shows better accuracy and stability. However, the calculation of the gradient of density and the correction matrix may further increase the computational cost. $^{67}$

\section{B. The motion of a damaged ship}

If the damage at the broadside is large for a damaged ship, the flow is mainly confined to the transverse section. Thus, the threedimensional motion of the ship hull can be simplified as a twodimensional flow problem around the cross section by ignoring the flow motion along the longitudinal direction. It should be pointed out that the roll motion coupled with other nonlinear effects is the most dangerous for a damaged ship in waves. Therefore, the strip theory is applied to study the dynamic response of the damaged ship in beam seas.

Strip theory was first proposed by Korvin-Kroukovsky, ${ }^{68}$ who made great contributions to the prediction of the wave load and ship motion. Based on the assumptions that the ship hull is a slender body and the disturbance of fluid flow along the longitudinal direction of the ship is negligible, the ship can be divided into several segments with a uniform sectional shape for each segment. Therefore, the fluid flow around the ship is simplified into a two-dimensional problem. In this study, the strip theory is introduced to develop the SPH numerical model. By integrating the hydrodynamics along the longitudinal direction of the ship, the internal and external fluid force on the whole ship can be obtained, and the coupling motion of roll-sway-heave can subsequently be calculated.

The motion in three degrees of freedom (DOF) is considered in this model, including roll, sway, and heave motions. The coupling of the internal and external flow field in each segment is considered according to Eqs. (2)-(4); the total dynamic force on the damaged ship is obtained by the sum of the force on each segment, ${ }^{68}$

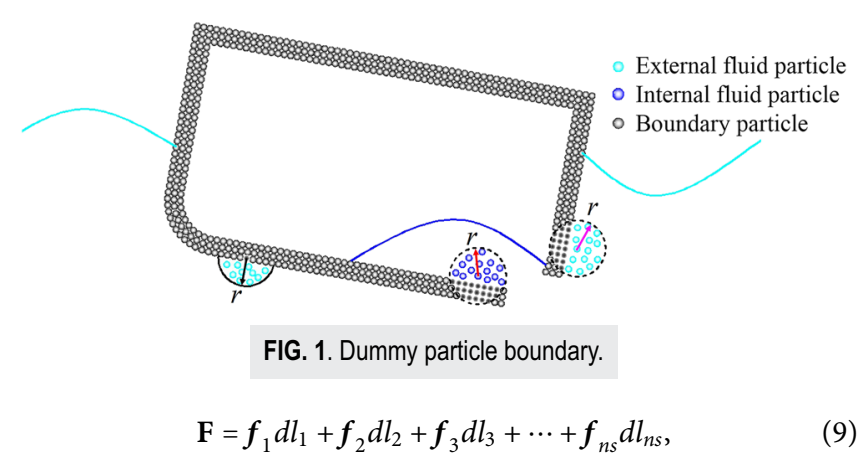

where $\boldsymbol{F}$ represents the total force on the floating body, $\boldsymbol{f}$ denotes the force per unit length of each segment, $d l$ is the length of each segment, and $n s$ is the number of segments.

The velocity and acceleration of the damaged ship model are calculated by the following equation: ${ }^{44}$

$$
\begin{gathered}
M \frac{D \mathbf{V}}{D t}=\sum_{n s}\left(\sum_{j \in B} \boldsymbol{f}_{j}\right) d l+M \mathbf{g}, \\
I \frac{D \boldsymbol{\Omega}}{D t}=\sum_{n s}\left(\sum_{j \in B} \boldsymbol{r}_{j 0} \times \boldsymbol{f}_{j}\right) d l, \\
\mathbf{f}_{j}=-\frac{m_{j}}{\rho_{j}} \sum_{i \in f}\left(P_{i}+P_{j}\right) \nabla W_{i j} \frac{m_{i}}{\rho_{i}},
\end{gathered}
$$

where $V$ is the velocity at the cabin barycentre, $\Omega$ is the angular velocity, $\boldsymbol{r}_{j 0}$ is the position vector of the boundary particle with $j$ pointing to the centre of the mass, and $\boldsymbol{f}_{j}$ is the fluid force per unit length on particle $j . M$ is the mass of the damaged ship model, and $I$ is the moment of inertia. $f$ and $B$ represent fluid and boundary particles, respectively. When calculating the artificial viscous term $\boldsymbol{F}_{v}$ in Eq. (3), the interaction between the fluid particle and the dummy particle is not considered; ${ }^{56}$ therefore, a free-slip boundary condition can be implemented on the ship model.

The flooding process of the damaged ship in waves is a complex problem involving the internal and external fluid motion. Since the immersion boundary exists, the dummy particle method ${ }^{69,70}$ is adopted which is a simplification of the fixed ghost particle proposed by Marrone et al. ${ }^{37}$ Interaction of the internal and external particles of the damaged ship through coupled motion with the cabin is illustrated in Fig. 1. Three layers of particles are laid to model the damaged ship boundary and the wave tank wall. The relative position of the boundary particles does not change during the simulation. It is noted that the support domain of particles in the flow field includes fluid particles and boundary particles, avoiding the error caused by the truncation of the boundary. The support domains of the boundary particles contain only the interacting fluids particles. The pressure of the boundary particles is obtained by interpolating from the adjacent fluid particles as ${ }^{69}$

$$
P_{B}=\left\{\begin{array}{ll}
\sum_{f} V_{f} W_{B f}\left[\rho\left(m_{B} \boldsymbol{a}_{B} / m_{f}-\boldsymbol{g}\right) \cdot \boldsymbol{r}_{B f}+P_{f}\right] / \sum_{f} V_{f} W_{B f}, & \sum_{f} W_{B f}>1 e-6 \\
0, & \text { Others }
\end{array} .\right.
$$


Wavemaker

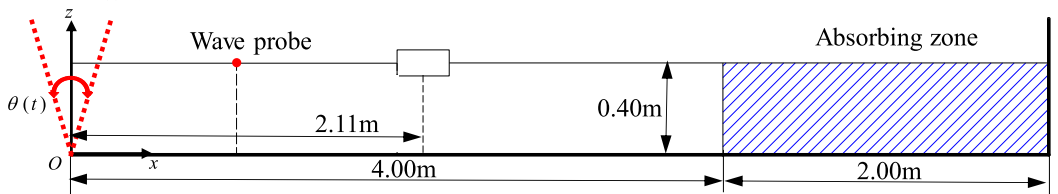

FIG. 2. Sketch of a floating box interacting with waves

The density of the boundary particles can be inversely solved by the Tait equation (4), ${ }^{54}$

$$
\rho_{B}=\rho_{0}\left(\frac{P_{B} \gamma}{c_{f}^{2} \rho_{0}}+1\right)^{1 / \gamma} .
$$

The acceleration of the floating body particles $\boldsymbol{a}_{B}$ is calculated according to the rigid body kinematics

$$
\boldsymbol{a}_{B}=\frac{D \mathbf{V}}{D t}+\frac{D \boldsymbol{\Omega}}{D t} \times \mathbf{r}_{B 0}+\boldsymbol{\Omega} \times \boldsymbol{\Omega} \times \boldsymbol{r}_{B 0} .
$$

\section{Numerical wave tank}

In the numerical wave tank, two ways are usually used to generate waves. One is to set the speed entrance at the boundary, and the other is to use a wavemaker. ${ }^{22,44}$ In this paper, a flap type wavemaker is adopted at one end of the wave tank. The height of the wave is controlled by the amplitude of the wavemaker, and the wavelength and period are controlled by the frequency of the wavemaker. ${ }^{71}$ The input signal to wavemaker is sinusoidal, and the regular waves are produced by the periodical motion of the wavemaker, as shown in Fig. 2. The angular motion ${ }^{71}$ of the wavemaker is

$$
\theta(t)=\theta_{0} \sin \omega t
$$

where $\theta_{0}$ is the amplitude of the flap wavemaker, $\omega$ is the frequency, and the period of the wavemaker is $T=2 \pi / \omega$, which is the same to the generated wave $T$. Then the amplitude of the flap wavemaker at the depth $z$ is

$$
a(z)=z \tan \theta(t) .
$$

The regular sinusoidal wave can be generated by the wavemaker under this motion.

When the wave travels to the boundary, they will be reflected. ${ }^{72}$ The reflected wave can subsequently disturb the incident wave and change the physical characteristics. Therefore, it is necessary to reduce the effects of reflected waves. To achieve that, a $\mathrm{PML}^{48,49}$ absorbing boundary condition is applied at the end of the wave tank. The PML method is practically implemented by adding a sink or a source term $\boldsymbol{D}_{v i}=-\sigma\left(x_{i}\right)\left(\boldsymbol{v}_{i}-\boldsymbol{v}_{0}\right)$ to the momentum equation of fluid dynamics [Eq. (3)]; the spatial function $\sigma\left(x_{i}\right)$ is suggested by Modave et al. ${ }^{73}$

$$
\sigma\left(x_{i}\right)=\left\{\begin{array}{ll}
\frac{c_{0}}{l} \frac{x_{i}-x_{0}}{\left(x_{0}+l\right)-x_{i}+d x}, & x_{0}<x_{i} \text { and } v_{x i}<0 \\
0, & x_{i} \leq x_{0} \text { or } v_{x i}>0
\end{array}\right. \text {, }
$$

where $x_{0}$ is the starting position, $l$ is the length of the absorbing zone, and $d x$ is the initial particle spacing. The velocity at the starting position of the absorbing zone $v_{0}=0.0$ is chosen in all simulations.

\section{NUMERICAL VALIDATION}

The present SPH model is verified against the experimental results $^{44,74}$ for the wave loads and the motion of a rigid floating body in waves. The efficiency of the absorbing zone responding to the wave loads in Sec. II is then examined in detail.

\section{A. The responses of a rigid body in waves}

The sketch of a floating box interacting with waves is shown in Fig. 2. Since the absorbing zone is added at the end of the wave tank, the length of the wave tank was reduced from $8.00 \mathrm{~m}$ in the experiment $^{44,74}$ to $6.00 \mathrm{~m}$. The rotation centre of the wavemaker is located at the origin of the coordinate, the depth of the tank is $h=0.40 \mathrm{~m}$, and the floating body is located at $x=2.11 \mathrm{~m}$ away from the flap wavemaker, which has a density of $\rho=680 \mathrm{~kg} / \mathrm{m}^{3}$ and an inertia moment of $I=14 \mathrm{~kg} \mathrm{~cm}^{2}$. The dimension of the floating body is $0.10 \mathrm{~m} \times 0.05 \mathrm{~m}$, and the input signal of the flap wavemaker is the same as the motion given in the experiment. ${ }^{74}$

The wave elevation at $x=1.16 \mathrm{~m}$ away from the wavemaker is shown in Fig. 3. The SPH results agree well with the experimental measurements. It is noted that the amplitude of the generated wave is close to the height of the floating body resulting in significant interaction of wave and the floating body.

The free-surface deformation and the position of the floating body are shown in Fig. 4 at two time instants of $t=7.26 \mathrm{~s}$ and $t=7.66 \mathrm{~s}$. Since the wavelength is much longer than the length of the floating body, the buoyancy of the floating body is approximately perpendicular to the wave surface, and the floating body moves in a

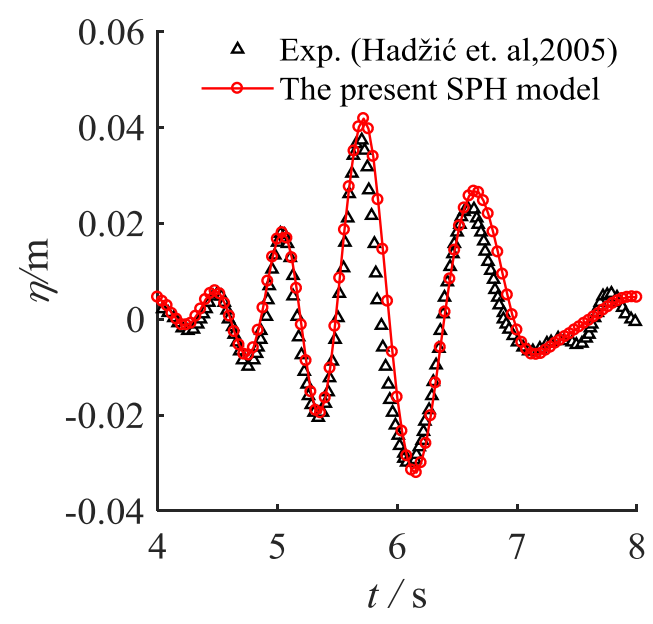

FIG. 3. Time history of the wave elevation at $x=1.16 \mathrm{~m}$ : comparison of the present $\mathrm{SPH}$ solution and experimental measurements. ${ }^{74}$ 


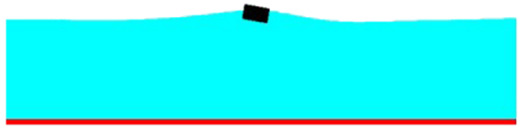

(a)

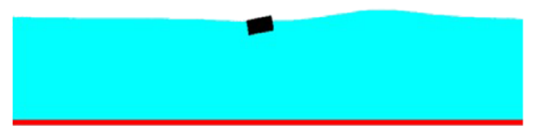

(b)
FIG. 4. Free surface deformation and position of the floating body at (a) $t=7.26$ and (b) $t=7.66 \mathrm{~s}$. circular orbit near the equilibrium position. At $t=7.26 \mathrm{~s}$, the amplitude of roll motion reaches the maximum, and the floating body is located at the peak of the wave; at $t=7.66 \mathrm{~s}$, the floating body is located at the trough, and the roll angle of the floating body reaches the reverse maximum.

Figure 5 shows a good agreement of the present numerical results and experimental measurements ${ }^{74}$ on centroid trajectories of the floating body. Despite the discrepancy noted at the later stage of floating body motion due to the strong nonlinear interactions, it is still a good demonstration that the present SPH model predicts well the wave motion and the interaction between the floating body and waves.

\section{B. Non-reflection of the wave tanks}

In order to validate the SPH model further in the prediction of the incident wave loads and the efficiency of the absorbing zone, the regular wave tank is modeled as illustrated in Fig. 6. The flap wave maker rotates around the origin of $x \mathrm{Oz}$. The wave tank has a depth of $H=30.0 \mathrm{~m}$ and a length of $L=5 H$. The length of the absorbing zone is $l=2 H$, approximately equaling to one wavelength. The angular motion of the flap wavemaker is

$$
\theta(t)=0.025 \sin 1.0 t
$$

then, a sinusoidal wave $\eta=A \sin (\omega t)$ with a wave amplitude of $A$ $=1.0 \mathrm{~m}$ and a period of $T=6.28 \mathrm{~s}$ is excepted to be generated. The wave height probes $\mathrm{WP}_{1}, \mathrm{WP}_{2}$ are located at $H$ and $2 \mathrm{H}$ away from the wavemaker. The comparison between the numerical prediction and the theoretical solution is shown in Fig. 7. The consistency is

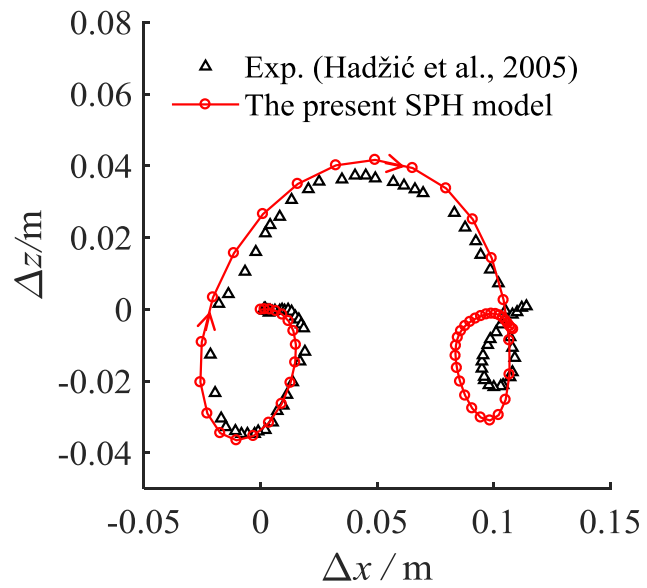

FIG. 5. Comparison of centroid trajectories of the floating body: SPH solutions vs. experimental measurements. ${ }^{74}$ also demonstrated in Fig. 7 with particle spacings of $d x=0.1 A$, $d x=0.2 A$, and $d x=0.4 A$.

The numerical results in Fig. 7 are shown in good agreement with the theoretical solutions, and the incident wave energy is absorbed effectively by the absorbing zone. The wave height on the probe is stable, indicating that the reflected wave is minimum. Therefore, the wave tank is used in the following simulation with the damaged ship model being located at $2 \mathrm{H}$ away from the wavemaker.

\section{RESULTS AND DISCUSSIONS}

\section{A. Numerical model}

Figure 8 shows the sketch of a damaged ship model in a wave tank. There are three cabins in the ship model which are in the same length $d l$ with a cross section of $U$-shape. The damaged ship model has a length of $L_{s}=3 d l=46.0 \mathrm{~m}$, a breadth of $B=16.0 \mathrm{~m}$, a depth of $D$ $=8.5 \mathrm{~m}$, and a draught of $d=4.2 \mathrm{~m}$, similar dimensions to a real ship. The middle cabin has an opening, and the empty cabin volume is $V$. The length of the opening is equal to the length of the middle cabin, and the height from the top of the opening to the bottom of the cabin is $\delta$, which is normalized with the draught of the ship model, namely, $\bar{\delta} \sim \delta / d$, if the opening facing the incident wave is marked as $\bar{\delta}^{-}$. The other two cabins are filled with liquid, and the loading ratio is defined as $\eta=h_{w} / D\left(h_{w}\right.$ denotes the height of the liquid cargo within the cabin). There are two wave height probes $x=1 / 160 B$ away from the cabin wall of the damaged side, W1 in a liquid cabin and W2 in the damaged cabin. The natural frequency of the ship model rolling without liquid loading is $\omega_{\phi}=1.06 \mathrm{rad} / \mathrm{s}$.

For all the following cases, the openings are under the waterline. It is noted that, compared with the total mass of the ship, the weight loss caused by the opening is small. Therefore, the total mass and gravity centre of the intact and the damaged ship model remain the same in the simulation. The damaged ship model is located at $2 \mathrm{H}$ away from the flap wavemaker. The moment the wavemaker begins to move is regarded as $t=0$. It is assumed that the cabin damage occurs at $t_{1}=3.18 T$ ( $T$ being the period of the incident wave) suddenly. At this time, the first complete wave has just propagated the distance $2 \mathrm{H}$ away from the wavemaker and the waves have been fully developed with a steady wave height. The uniform particle distribution is adopted here, and the particle spacing is $d x=$ $0.1 \mathrm{~A}$. For the symmetry, only two segments are needed to simulate, that is, the intact cabin segment with or without liquid and the damaged cabin segment. The simulation cases are listed in Table I, and all cases are under a regular beam wave with a wave amplitude of $\bar{A}=10 / 21$ which is normalized by the draught $d$ and a wave angular frequency of $\omega_{w}=1.0 \mathrm{rad} / \mathrm{s}$, which is close to the natural roll frequency of the ship $\omega_{w} / \omega_{\phi}=0.94$. The non-dimensional frequencies of the ship motion and sloshing are listed in Table II, where the frequencies are normalized by the wave frequency $\omega_{w}$. The sloshing frequency in the U-shape tank is calculated by ABAQUS. ${ }^{75}$ 

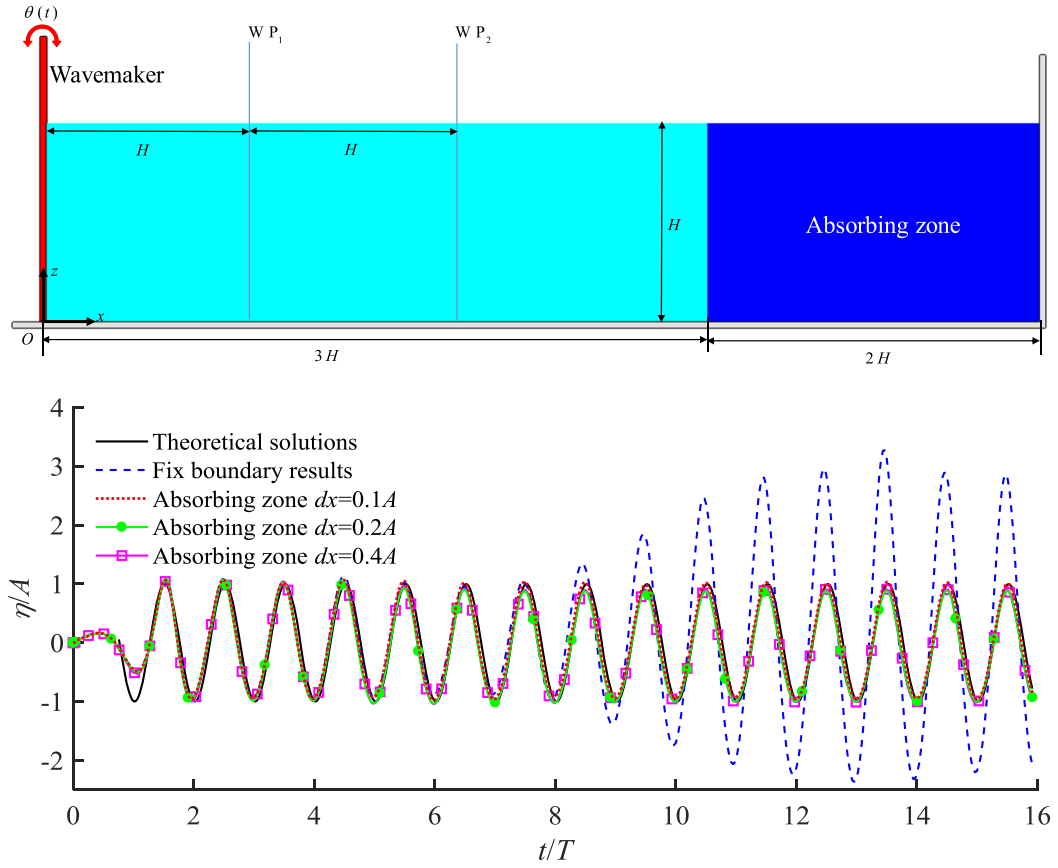

(a)

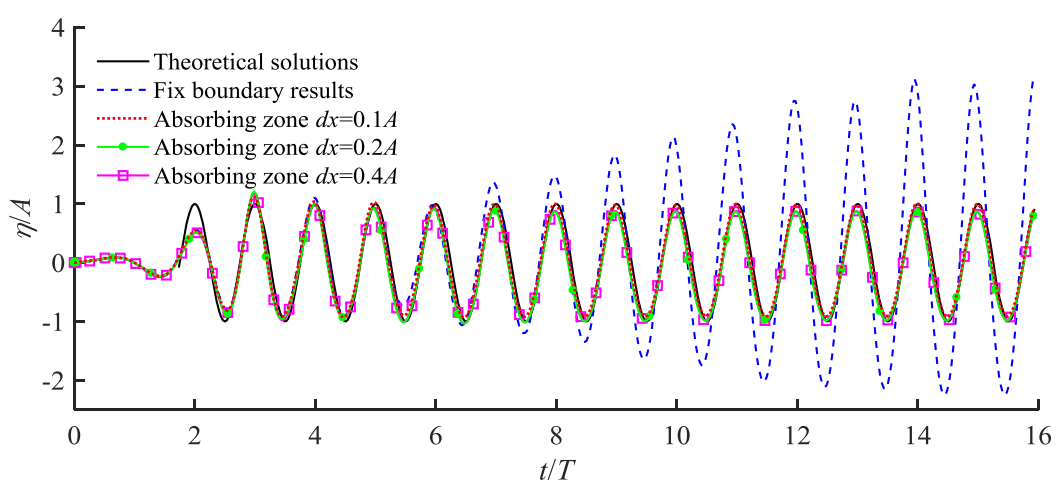

(b)
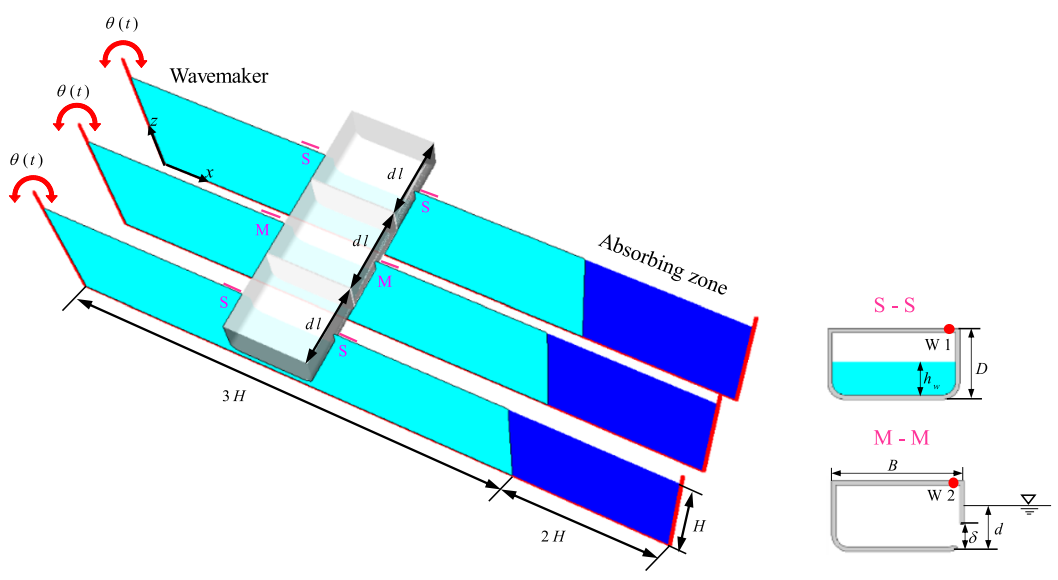

FIG. 6. The sketch of the wave tank.

FIG. 7. Wave heights measured at the different probes: (a) $W P_{1}$ and (b) $W P_{2}$.

FIG. 8. The sketch of a damaged ship in a wave tank. 
TABLE I. Simulation case matrix.

\begin{tabular}{lrl}
\hline \hline No. & Opening size $\bar{\delta} \sim \delta / d$ & Liquid ratio $\eta=h_{w} / D(\%)$ \\
\hline Case 1 & $\bar{\delta}=0$ & $\eta=0$ \\
Case 2 & $\bar{\delta}=5 / 21$ & $\eta=0$ \\
Case 3 & $\bar{\delta}=10 / 21$ & $\eta=0$ \\
Case 4 & $\bar{\delta}=5 / 7$ & $\eta=0$ \\
Case 5 & $\bar{\delta}=10 / 21$ & $\eta=0$ \\
Case 6 & $\bar{\delta}=10 / 21$ & $\eta=25$ \\
Case 7 & $\bar{\delta}=10 / 21$ & $\eta=50$ \\
Case 8 & $\bar{\delta}=10 / 21$ & $\eta=75$ \\
\hline \hline
\end{tabular}

\section{B. The effect of opening sizes}

The dynamic response of a damaged ship in waves is affected by many factors, so the effect of opening sizes is first studied in this section. The three different dimensionless opening sizes are chosen: $\bar{\delta}=5 / 21, \bar{\delta}=10 / 21$, and $\bar{\delta}=5 / 7$. The liquid ratio in the two intact cabins is $\eta=0 \%$. The damage to the middle cabin is assumed to occur at $t_{1}$ suddenly. The cabin is free to move under wave loads before it is damaged.

Figure 9 shows the internal and external velocity fields of the damaged ship with an opening of $\bar{\delta}=10 / 21$. The damaged ship rolls to the opposite side of the opening primarily due to the actions of the waves and the gravity of the cabins at the initial stage after the damage occurs. A phase shift is observed between the liquid sloshing inside the damaged cabin, the rolling of the ship, and the external waves in Fig. 9(a). As the flooding process continues, the wave profile outside the cabin is relatively stable, while the sloshing process inside the cabin exhibits significant nonlinearity featured by splashing, jetting, and crushing, as shown in Fig. 9(b). With the increase in the flooding water, the sloshing wave with a certain amplitude inside the cabin forms, which has a phase difference from the external waves, as plotted in Figs. 9(c) and 9(d). Due to the coupling effects, the phase shift and the amplitude of roll motion gradually reduce [see in Fig. 9(e)]. Additionally, with the exchange of the inflow and outflow at the opening, it can be observed that the flooding water, the cabin, and the external wave tend to oscillate at a same pace.

For better understanding of the dynamic flooding process, the roll motion, heave motion, and the flooding water accumulation of the damaged ship with an opening of $\bar{\delta}=10 / 21$ in still water and waves with the wave amplitude of $\bar{A}=5 / 21, \bar{A}=5 / 14$, and $\bar{A}=5 / 7$ are shown in Fig. 10. When the ship damage occurs in still water, the ship first rolls to the opening and the maximum roll angle occurs on the opposite of the opening. The middle cabin is damaged suddenly at the instant of $t_{1}$. As shown in Fig. 10(a), the incline
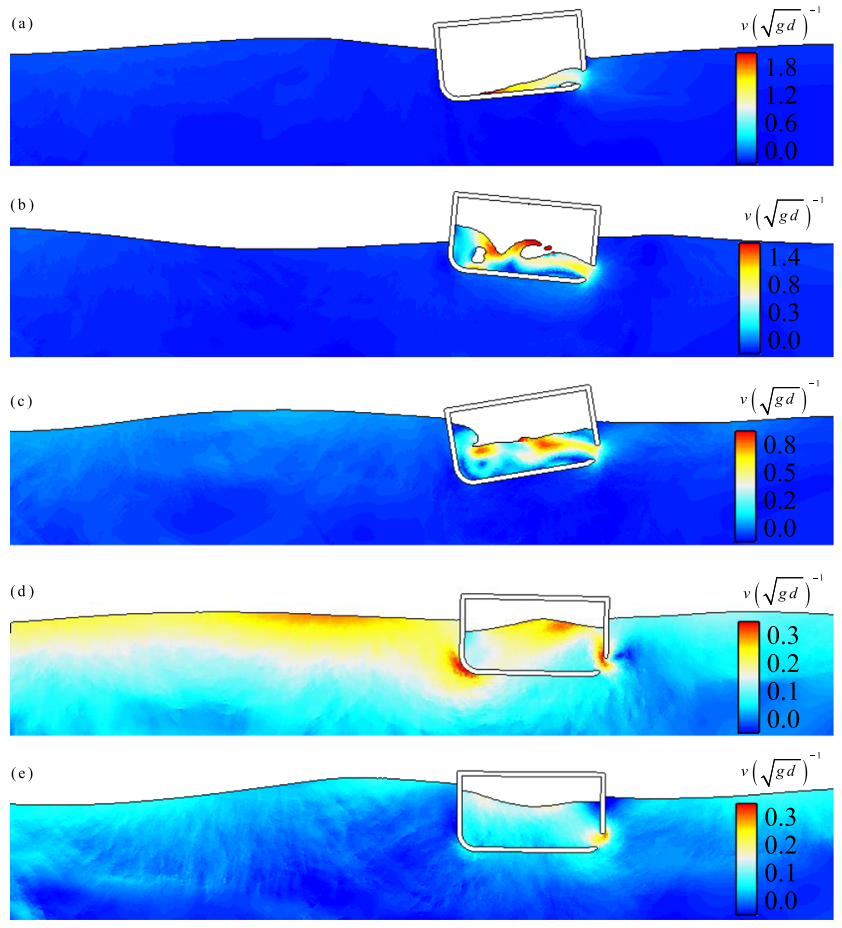

FIG. 9. The motion of the damaged cabin with an opening of $\bar{\delta}=10 / 21$ under combined internal liquid and external wave loads at (a) $t=3.42 T$, (b) $t=4.00 T$, (c) $t=4.46 T$, (d) $t=6.40 T$, and (e) $t=6.57 T$.

moment induced by the flooding water and the external wave act in opposite directions on the ship at the initial stage after the damage occurs. Consequently, the inflow rate slows down compared to the still water case, as shown in Fig. 10(c). When the flooding water accumulation reaches the first peak, the amplitude of the roll motion of the ship reduces significantly, and the moment induced by the flooding water is dominant until the flooding water and the external wave tend to oscillate at a same pace, as observed in Fig. 9(e). For the damaged ship with the same opening in different wave amplitudes, the newly established equilibrium position of heave motion is the same, and the amplitude of heave motion is mainly determined by the amplitude of the wave.

The velocity fields of the damaged cabin with different opening sizes are shown in Fig. 11. After the damage occurs to the cabin, the external water flows in through the opening, rushes to the opposite side of the opening, and climbs along the wall, as shown in Fig. 11(a).

TABLE II. Non-dimensional frequency of the ship motion and sloshing.

\begin{tabular}{lccc}
\hline \hline Name & The natural frequency & Case (\%) & The first natural sloshing frequency \\
\hline Roll motion of the ship & $\bar{\omega}_{\phi}=1.06$ & $\eta=25$ & $\bar{\omega} 1_{0}=0.87$ \\
Heave motion of the ship & $\bar{\omega}_{H}=1.28$ & $\eta=50$ & $\bar{\omega} 2_{0}=1.12$ \\
Wave frequency & $\bar{\omega}_{w}=1.00$ & $\eta=75$ & $\bar{\omega} 3_{0}=1.26$ \\
\hline \hline
\end{tabular}




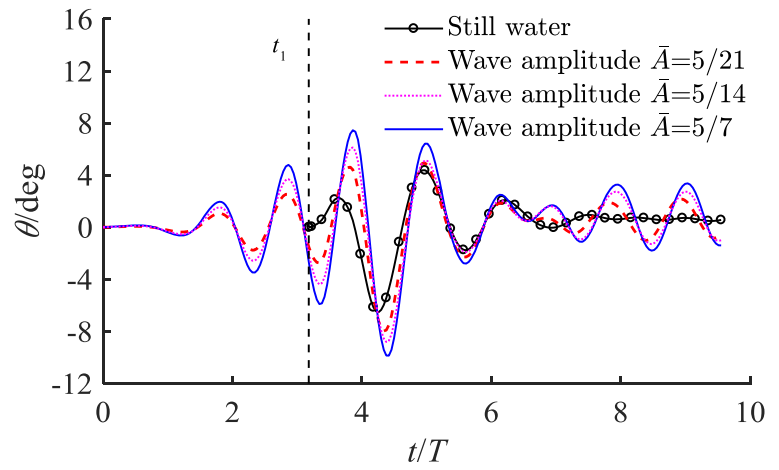

(a)

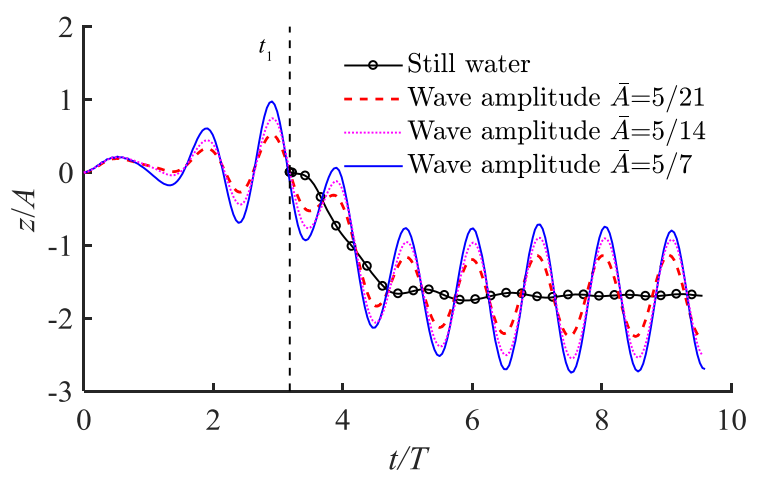

(b)

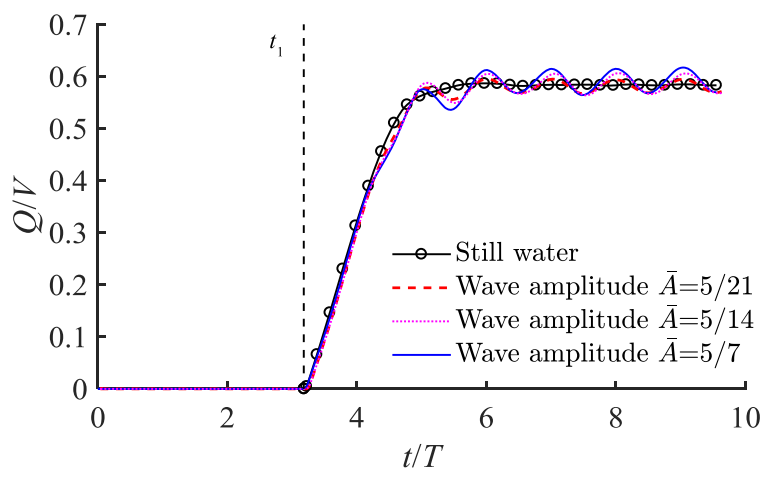

(c)

FIG. 10. The (a) roll motion, (b) heave motion, and (c) flooding water accumulation of the damaged ship in waves with different wave amplitudes.

It is clearly evident that a cavity formed near the bilge due to a high-speed overturning of the flooding water. The climbing height of the water and the cavity size increase with the increase in the opening size [see in Fig. 11(b)]. In addition, a water jet is generated due to the water impact on the internal free surface, which can further lead to more complex flow phenomena due to the breaking and splashing of the free surface inside the cabin. It is seen that more water is flooded into the cabin and it sinks more quickly for the case of a larger opening. With the increase in the flooding water, the violent sloshing takes place in the cabin and the water appeared even to reach the deck for the case of the large opening $(\bar{\delta}=5 / 7)$, as shown in Fig. 11(c). As the water accumulates in the cabin, a traveling sloshing wave inside the cabin is formed due to the combined excitation of the cabin motion and the external wave force. Since the water sloshing inside the cabin introduces damping effects on the rolling of the cabin, consequently, the rolling amplitude reduces more for the larger opening case. As shown in Fig. 11(d), the sloshing wave height inside the damaged cabin with the smaller opening is larger than that for the case with a larger opening. In order to demonstrate this further, surface elevations at the location of probe W1 inside the damaged cabin are presented in Fig. 12. The surface elevation in the damaged cabin increases immediately following the damage occurrence. The elevation of the surface on the probe increases with the opening size increasing at the initial stage $\left(t_{1}<t / T<5\right)$. However, due to the violent sloshing damping effects, the height of the sloshing wave reduces with the increase in opening size. When flood water in the cabin reaches a dynamic balance [Fig. 11(e)], the coupling effects of the flooding water, the cabin, and the waves are more significant. The sloshing wave surfaces inside the damaged cabin are seen approximately matching their respective external ones, and the exchange of the inflow and outflow at the opening still takes place with a certain velocity.

Figure 13 shows the flooding water accumulation of the damaged cabin with different opening sizes in waves, and the flooding amount $Q$ is normalized by the empty cabin volume $V$. It is seen that the damage to the ship occurs at $t_{1}$ and the water floods the cabin quickly. The amount of the flooding water increases approximately linearly with time at the initial stage. However, with the flooding water accumulating and exchanging at the opening, the inflow rate slows down. Since the position of the opening is below the waterline, the opening is alternately lifted and dropped when the wave crest and trough pass the cabin. Accordingly, the periodical inflow and outflow are induced, and the inflow and outflow rates reach a dynamic balance at $t_{2}$. It should be pointed out that an approximately uniform $t_{2}$ is marked and chosen for the description; however, clear observation in Fig. 13 reveals that the time taken for the damaged ship to reach its dynamic balance for the damaged cabin with different opening sizes is different. The variation of the flooding amount is close to $10 \%$ of the maximum amount of flooding water inside the damaged cabin. For the small opening size of $\bar{\delta}=5 / 21$, as clearly evident in Fig. 13, there is a short stagnation period in the flooding process before the first peak of the flooding amount. A counterclockwise vortex [see in Fig. 13(b)] formed near the opening due to the coupling effect between the flooding water, ship hull, and waves, which prevents the inflow. However, after the wave passes, the cabin continues to flood under the incline moment. With the increase in opening sizes, the peak of the flooding amount at the initial stage increases and the time taken to reach the dynamic equilibrium reduces but tend to reach a stable oscillation pattern after a short time for all three different openings.

The heave motions of the damaged cabin with different opening sizes in waves are shown in Fig. 14. The damaged ship is seen undergoing a periodic heave motion near an equilibrium position. As can be seen, the amplitude and period of heave motion are close to those of the intact ship model before $t_{1}$, when the damage to the cabin occurs. From $t_{1}$ to $t_{2}$, the damaged cabin begins 

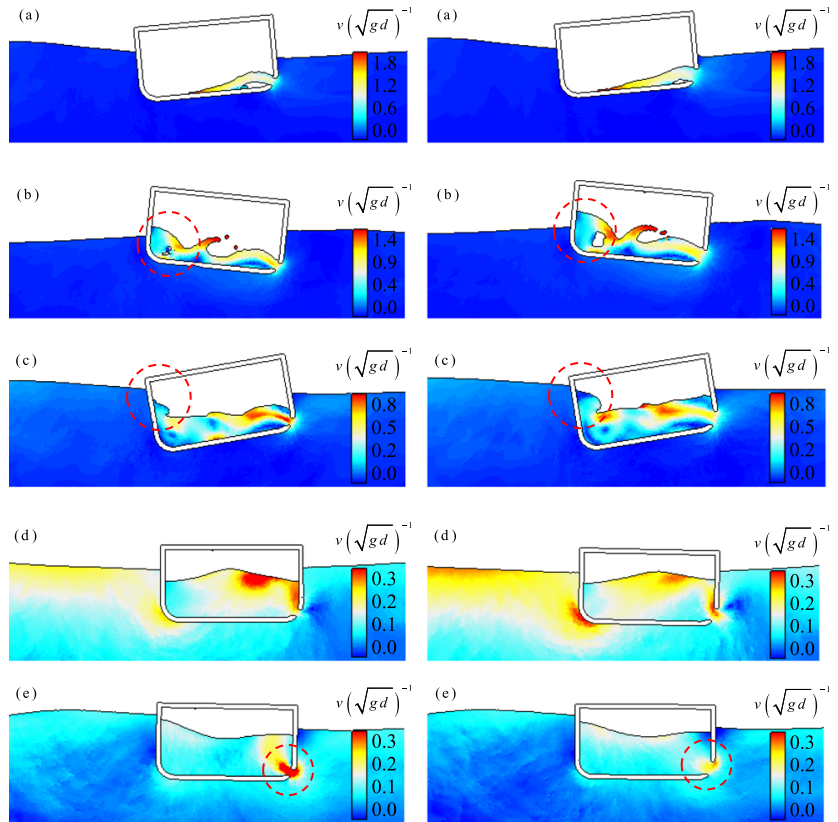

(1) $\bar{\delta}=5 / 21$

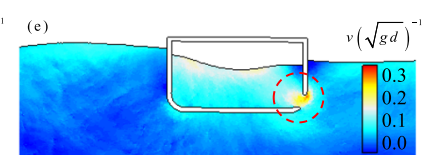

(2) $\bar{\delta}=10 / 21$

to flood and losses its equilibrium. The barycentre of the damaged cabin shows a significant drop, and the larger the opening size, the lower the barycentre. Additionally, the period of the heave motion becomes slightly larger due to the damping effect of the flooding water inside the cabin. With the accumulation of the flooding water, the heave motion of the cabin with different opening sizes tends to be consistent and oscillates around the newly established equilibrium position. Beyond $t_{2}$, the heaving behavior of the damaged ship is similar to the intact ship in waves around their equilibrium positions, respectively.

The roll motions of the damaged ship with different opening sizes and the intact ship are shown in Fig. 15, where the angle to the wave propagation direction is positive, and vice versa. It can be seen that the ship rolls with a small amplitude under the wave impact before $t_{1}$, but the amplitude increases gradually. At the instant of $t_{1}$, the middle cabin is damaged suddenly. The damaged ship begins to

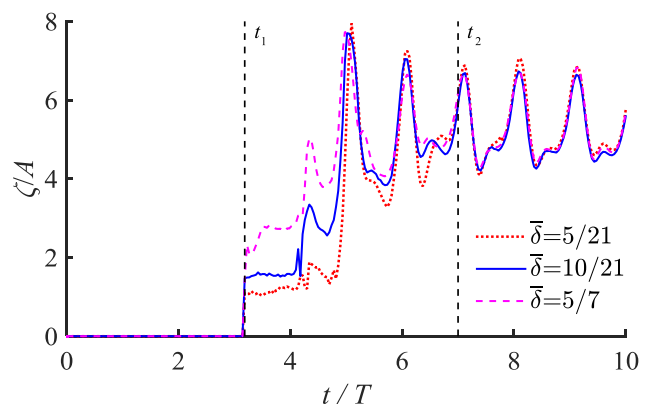

FIG. 12. Surface elevations at the location of probe W1 in the damaged cabin with different opening sizes in waves.
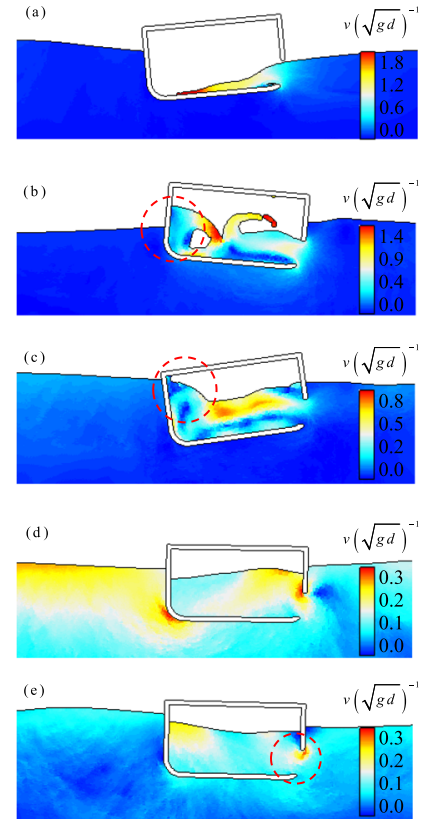

(3) $\bar{\delta}=5 / 7$

flood and first rolls to the opposite side of the opening. Because of the negative effects of the incline moment induced by the flooding water on the wave moment, the rolling amplitude is reduced slightly compared to that of the intact one. In the transient flooding stage, the rolling amplitude continues to increase until $t^{\prime}$, where a sharp reduction in rolling amplitude occurs. The damaged ship with different openings shows a significant reduction in rolling amplitude, due to the damping effect introduced by the additional free surface in the damaged cabin, while the rolling amplitude of the intact ship continues to increase. It indicates that the incline moment induced by the flooding water and external wave are dominant effect factors. For $t^{\prime}<t / T<t_{2}$, the flooding water accumulation reaches the first peak; the moment induced by the inflooding water takes the dominant effect on the ship roll. The oscillations of the damaged ship for all three openings appeared to be significantly reduced until a stable status $\left(t_{2}\right)$ has been reached in which the damaged ship rolls at approximately the same amplitude and period. However, a slight difference in the onset of stable roll motion is observed in Fig. 15.

\section{The effect of incident wave direction}

The coupling of internal and external flow field can affect the velocity distribution near the opening and subsequently affect the flooding process. Thus, the relative position of the incident wave direction and the opening is examined. The middle cabin is damaged with an opening $\bar{\delta}=10 / 21$ at the time of $t_{1}$. It is defined as the head wave (marked as $\bar{\delta}^{-}=10 / 21$ ) when the opening is facing the incident wave; otherwise, it is defined as the back wave (marked as $\bar{\delta}=10 / 21)$.

Figure 16 shows the horizontal velocity distribution of the flow field with the opening under the head wave and back wave, respectively. In the head wave condition with the opening of $\bar{\delta}^{-}=10 / 21$, 


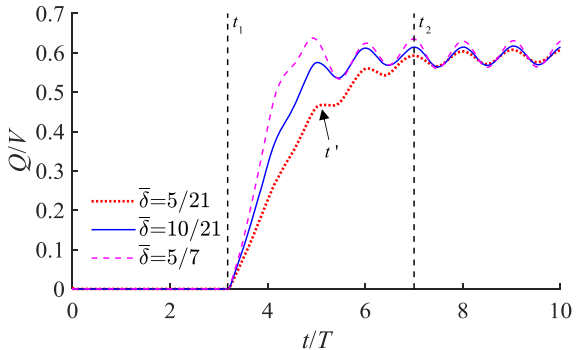

(a)

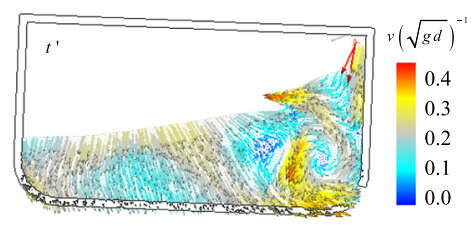

(b)

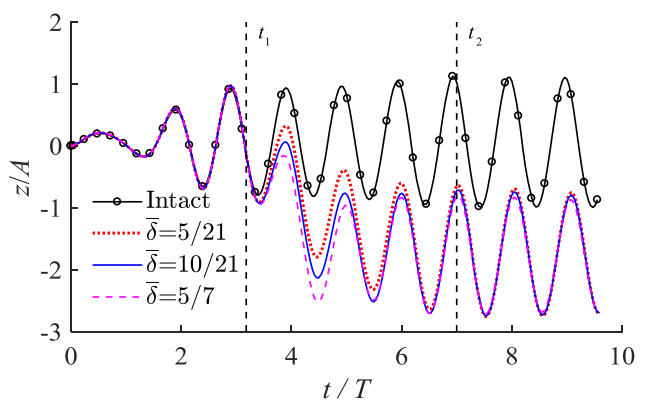

FIG. 14. Heave motions of the damaged cabin with different opening sizes in waves

it is seen that the wave first pushes the water to flow through the opening. A flooding jet with a larger velocity is formed and rushes to the wall of the cabin rapidly opposite to the opening. The upward flow is seen reaching higher than that of the back wave condition, as can be seen in Fig. 16(a). Due to the positive superposition of the moment induced by flooding water and the wave moment, the initial rolling angle of the head wave case is larger. Conversely, the negative superposition takes place for the latter case and thus a smaller rolling angle occurs [see Fig. 16(b)]. As can be observed in Figs. 16(c) and 16(d), the flooding process exhibits an immediate change following the changes in the immersion depth of the opening. For the case of the head wave, the opening is lifted upwards leading to the flooding process slowing down, while the opening is immersed further for the case of the back wave. During this period, it is evident that the exchange is frequently formed near the opening. Finally, with the accumulation of flooding water, the flooding speed reduces and the amount of the flooding water gradually reaches a dynamic equilibrium, as shown in Fig. 16(e).
FIG. 13. (a) Flooding water accumulation of the damaged cabin with different opening sizes in waves. (b) The velocity vector of internal flooding water at $t^{\prime}$ of the damaged cabin with $\bar{\delta}=5 / 21$.

The effect of the incident wave direction on the amount of the flooding water is shown in Fig. 17. Before $t_{1}$, the cabin is not damaged, so the amount of the flooding water is zero. When the damage occurs at $t_{1}$ with an opening, the water begins to flood into the cabin with an increasing flow speed under ambient water pressure. At the initial stage after damage, the inflooding rate for the head wave case is larger than that for the back wave case until the exchange is formed near the opening [Fig. 16(c)]. As can be seen within the time span $t_{1}<t / T<5$, the amount of the flooding water approximately linearly increases. However, under the wave impact, the immersion depth of the opening changes and the flooding amount fluctuates when the wave crest and trough pass. Consistent with the observations made in Fig. 16, numerical calculation demonstrates that the amount of the flooding water for the head wave condition is less than the still water case due to the slowing down flooding process as the consequence of the opening is lifted up, while the opposite is true for the back wave case since the flooding speed is enhanced by the deeper opening (see Fig. 17).

The effect of the incident wave direction on the roll motion is shown in Fig. 18. The intact ship rolls under the wave impact before $t_{1}$, and the water begins to flood in when the opening is produced due to the damage occurring at $t_{1}$. At the initial stage, the wave direction and the flooding water flow direction are the same for the head wave case and the water flow in the cabin takes great effects; thus, the flooding speed is larger for the head wave case and consequently leads to a larger rolling angle than that for the back wave case (see for $t / T<5$ ). However, soon the wave force takes dominant effects on the roll motion. Due to the coupling of internal water sloshing and the wave impact, the rolling amplitude is gradually reduced because of the negative superposition of the moments and it is more obvious for the head wave case. It is seen that the roll motions show many periods

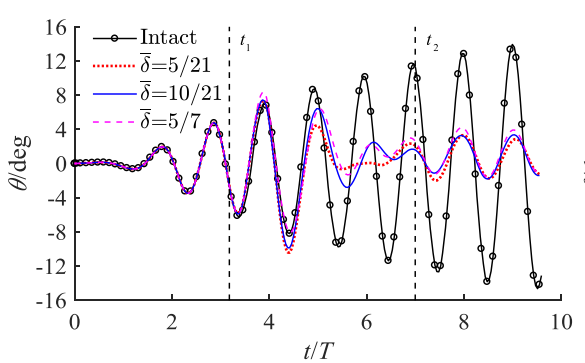

(a)

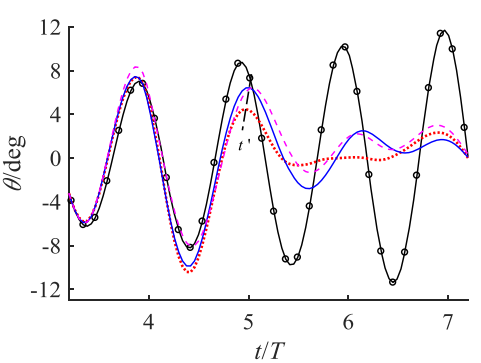

(b)
FIG. 15. (a) Roll motion of the ship with the damaged cabin of different opening sizes. (b) The snapshot between $t_{1}$ and $t_{2}$. 

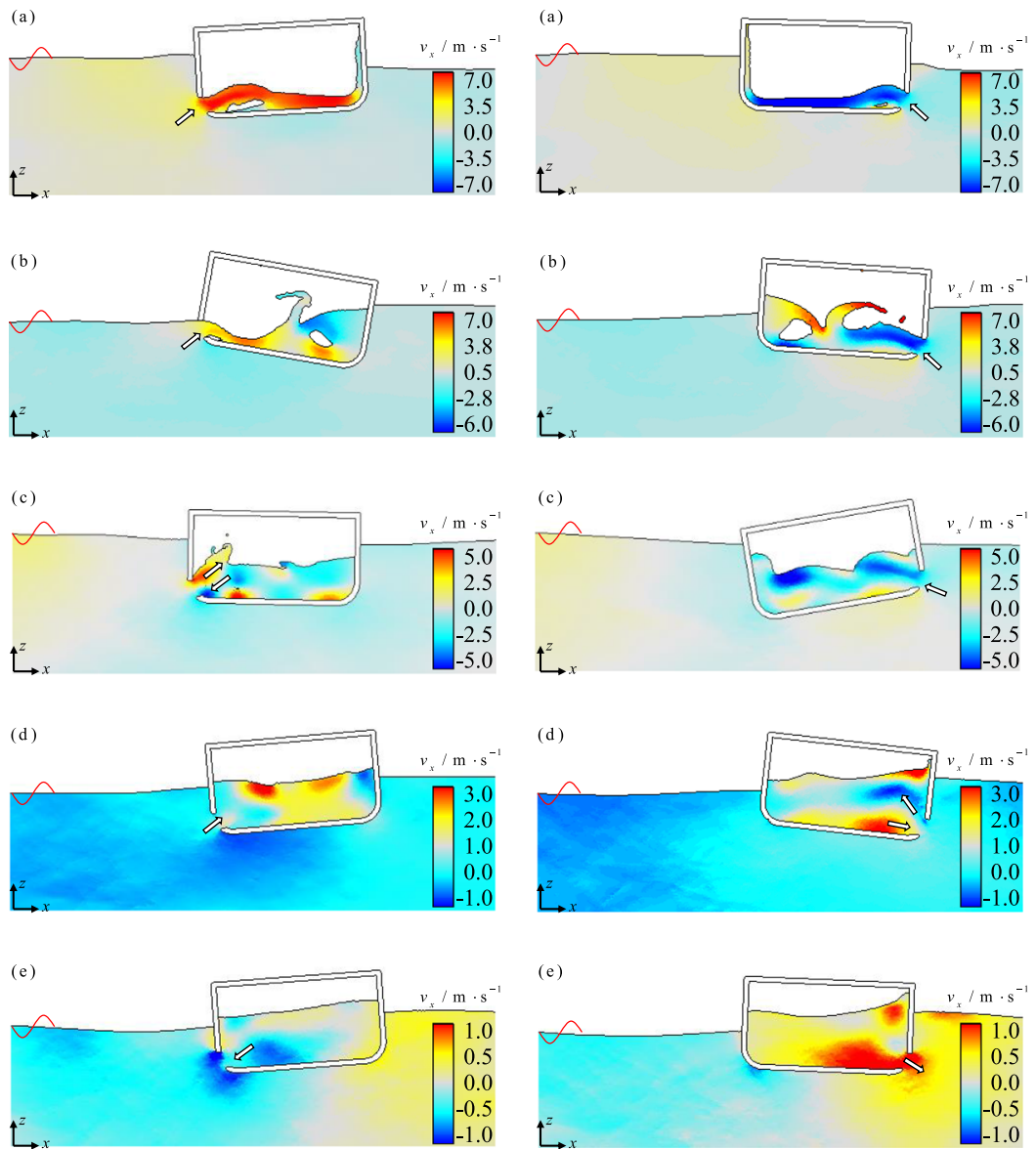

(1)

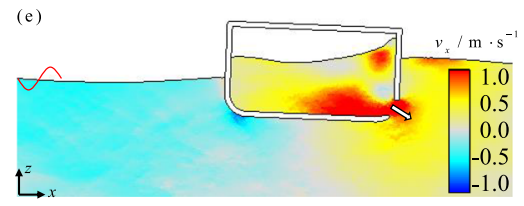

(2)
FIG. 16. The horizontal velocity distribution of flow field with opening $\bar{\delta}=10 / 21$ under head wave (1) and back wave (2), respectively, at (a) $t=3.62 \mathrm{~T}$, (b) $t=4.00 \mathrm{~T}$, (c) $t=4.38 \mathrm{~T}$, (d) $t=5.02 T$, and (e) $t=6.09 T$. with the head wave case exhibiting more severe oscillation for a short initial period but decreases more rapidly compared to the case for the back wave. These differences may be because the flooding induced moment takes dominant effects for the head wave case.

Figure 19 shows the effect of the incident wave direction on the heave motion of the damaged ship. Although the opening due to the damage occurrence to the cabin significantly alters the characteristics of the heave motion, the incident wave direction, however, has only a slight effect on the heave motion. The heave motions exhibit the wave frequency characteristics. As can be seen in Fig. 19, the heaving amplitude of the head wave case is slightly larger than that for the back wave case. This is attributed to the fact that the heave motion is mainly affected by the amount of the flooding water and the internal water sloshing, especially when the flooding process is slow. The internal water sloshing under head wave is more violent than that in the case of the back wave.

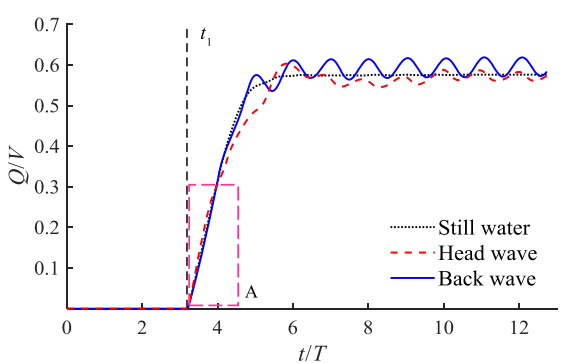

(a)

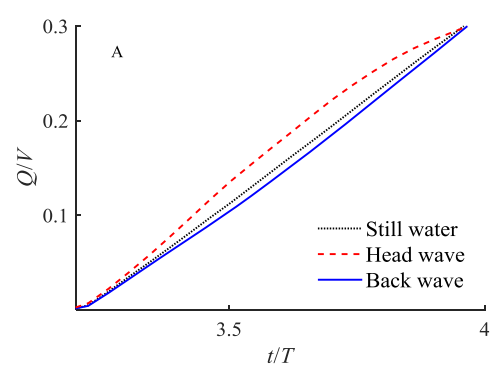

(b)
FIG. 17. (a) The effect of the incident wave direction on the amount of the flooding water. (b) A snapshot of the transient stage immediately after damage. 


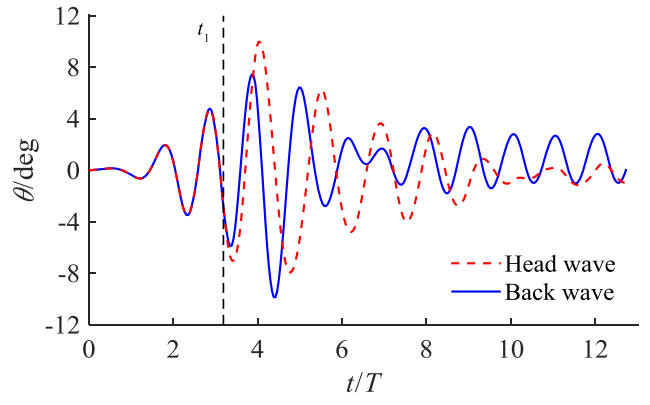

FIG. 18. The effect of the incident wave direction on the roll motion.

\section{The coupling effects of liquid cabins, flooding water, and the damaged ship}

When damage occurs to a ship in waves, the liquid sloshing inside the other undamaged cabins may further complicate the dynamic response of the damaged ship. The effect of different ratios of the liquid filled in the undamaged cabins is studied in this section. For a three cabin configuration, damage is assumed to occur to the middle cabin at the time of $t_{1}$ with an opening of a size $\bar{\delta}=10 / 21$ locating at the bilge, while the other two undamaged cabins are filled with a same volume of liquid. Different volumes filled in the liquid cabins are discussed: (1) the ratio $\eta=0 \%$; (2) the ratio $\eta=25 \%$ and

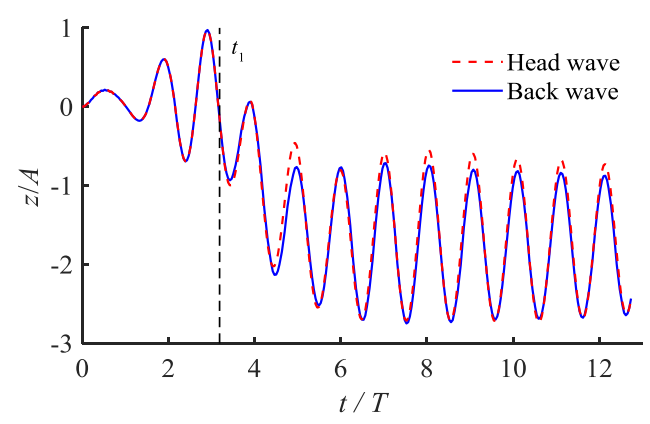

FIG. 19. The effect of the incident wave direction on the heave motion.

$\bar{\omega} 1_{0}=0.87 ;$; $(3)$ the ratio $\eta=50 \%$ and $\bar{\omega} 2_{0}=1.12$; and (4) the ratio $\eta$ $=75 \%$ and $\bar{\omega} 3_{0}=1.28$, where $\omega_{0}$ is the first natural frequency of the sloshing. In order to compare the effect of the filled liquid cabin, it is assumed that the ship models with different loading ratios have the same draught before the damage creation.

The coupling motion of the damaged ship when the two undamaged cabins filled with different ratios of liquid is shown in Fig. 20. It is seen that different rolling angles are produced at the initial stage due to the coupling effect of liquid sloshing [Fig. 20(a)]. Because the water flowing through the opening leads to the asymmetry of pressure loading on the ship surface, the roll motion is excited. However, due to different ratios of liquid filled in the cabin, under
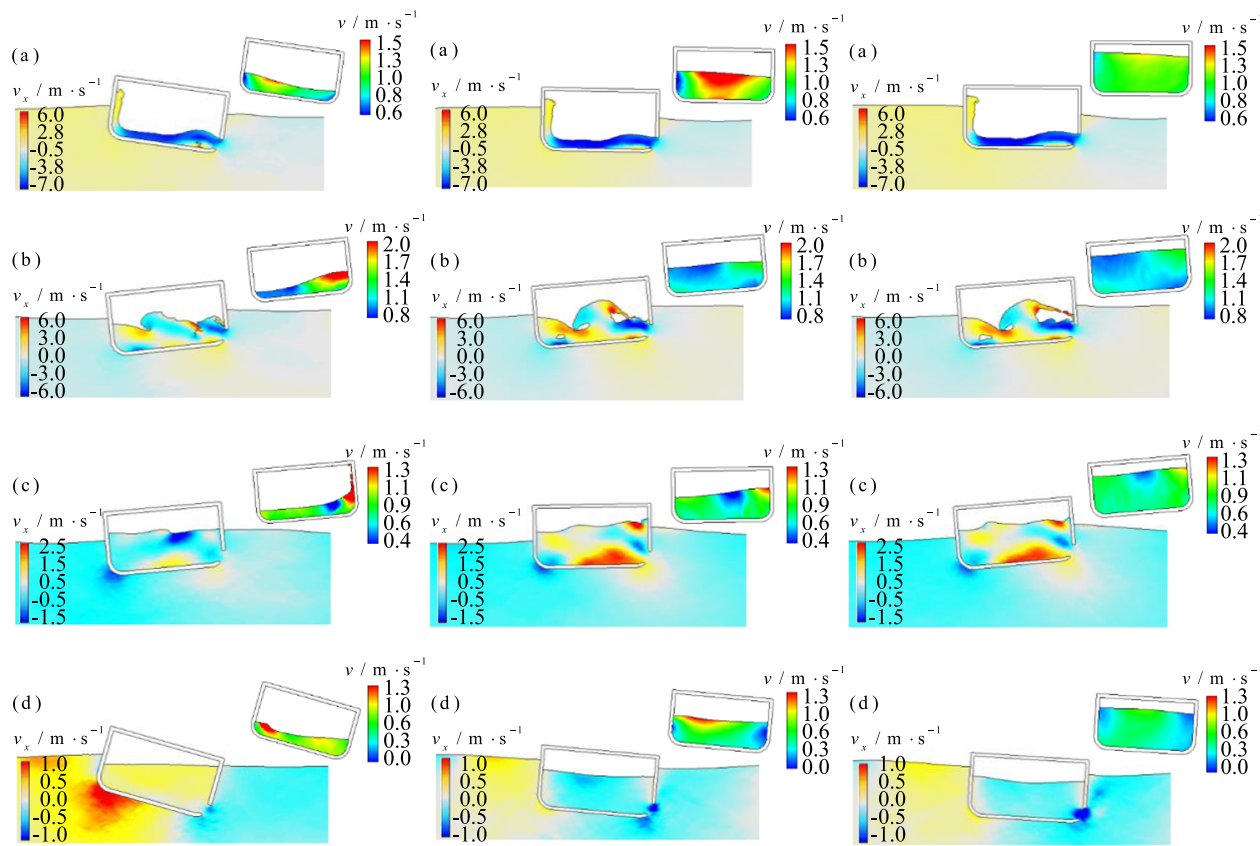

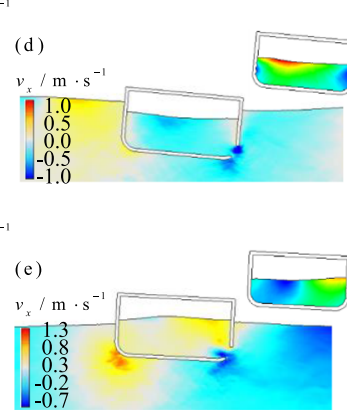

(2) $\eta=50 \%$
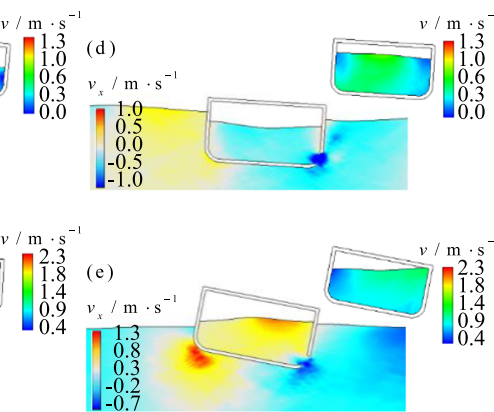

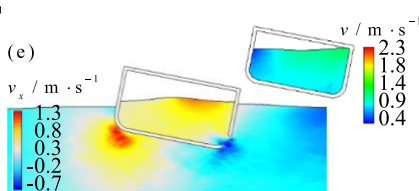

(3) $\eta=75 \%$
(1) $\eta=25 \%$

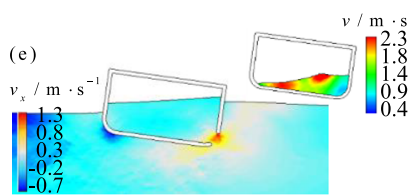

FIG. 20. The motion of the damaged ship with the two undamaged cabins filled with different ratios of liquid at (a) $t$ $=3.70 T$, (b) $t=4.22 T$, (c) $t=5.18 T$, (d) $t=8.80 \mathrm{~T}$, and (e) $t=10.15 \mathrm{~T}$. The figure at the top right corner shows the undamaged cabin. 
the same heeling moment, the damaged ship exhibits different heeling angles. There is a larger heeling angle for the liquid ratio of $\eta$ $=25 \%$ but a smaller heeling angle for the large ratios. Also, the liquid in the cabins shows different sloshing amplitudes and sloshing velocities [Fig. 20(b)]. Moreover, it is evident that the phase difference between the liquid sloshing and the water flooding takes place. The lower the filled liquid ratio is, the earlier the phase difference occurs, as seen in Figs. 20(b) and 20(c). Accordingly, the flooding water in the damaged cabin tends to reach stability earlier. In Fig. 20(d), the water velocities for the larger filled liquid ratios are still very large and sloshing violently compared to the case of the ratio of $25 \%$. However, with the accumulation of flooding water, most of the cabin is filled with water; a degree of consistency is reached between the sloshing of the flooding water and the liquid in undamaged cabins [Fig. 20(e)].

The roll motion of the damaged ship with two cabins filled with liquid in waves is shown in Fig. 21. Prior to the damage occurring at $t_{1}$, the intact ship is free to move under combined external wave exciting and internal sloshing loads. It is seen that the amplitude and period of the roll motion are reduced due to the internal liquid sloshing, especially for the cases of cabins with filling ratios of $50 \%$ and $75 \%$. The more the liquid filled in the cabins, the more pronounced such reduction is. When the middle cabin is damaged at $t_{1}$, the negative superposition of the heeling moments takes place for the moment generated by the liquid cabins and the moment from the flooding water in the damaged cabin. As can be seen in Fig. 21, the rolling angle decreases to a certain extent resulting from the negative superposition of the heeling moments when the liquid cabins filled with liquid. For the case with the filling ratio of $25 \%$, the transient flooding changes the rolling angle only slightly, but the rolling angle is seen decreasing significantly for the case with the filling ratio of $75 \%$. For $t / T>t_{2}$, when the amount of the flooding water reaches dynamic equilibrium, the natural rolling frequency of the damaged ship is rather different compared to that in previous stages $(t / T$ $<t_{2}$ ), and the effect of the liquid ratio becomes more pronounced. The rolling angle of the ship is no longer around zero instead the ship will roll around a new angle. Because the natural frequency of the system is composed of the flooding water in addition to the ship hull and the liquid cabins, the roll motion shows different features. For the case of the ratio of $25 \%$, the new equilibrium angle is shifted far from zero and the damaged ship experiences roll motion with a large amplitude and high frequency. But for the case with the fill ratio of $75 \%$, the new equilibrium angle is smaller than that for the case of $25 \%$ accompanied with smaller rolling amplitude and lower

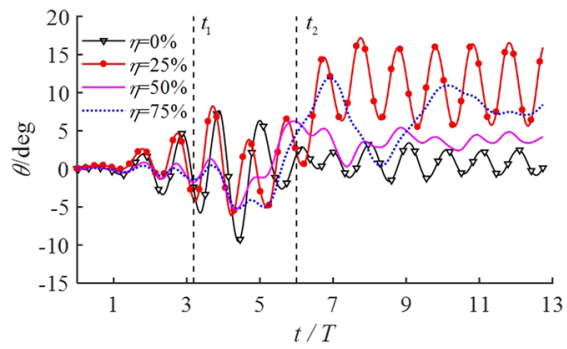

FIG. 21. The roll motion of the damaged ship partially filled with liquid in waves. frequency, because the sloshing frequency is far away from the wave. It is noted that the roll motion is rather stable with a small amplitude for the case with the filling ratio of $50 \%$. Thus, it can be demonstrated that there is a critical liquid filling ratio maximizing the damping effect to stabilize the ship.

The time histories of the surface elevations in the damaged cabin and liquid cabin, respectively, are shown in Fig. 22. The sloshing in the liquid cabins couples with the ship motion and the external wave excitation before the damage to the middle cabin at $t_{1}$, so the free surface within the liquid cabins is disturbed with a small amplitude being generated. When the middle cabin is damaged at $t_{1}$, the flooding water rushes into the cabin, leading to significant changes in the heeling moment. As a result, the amplitude of the sloshing wave in the liquid cabins decreases with the increase in the ratio of liquid filled in the cabins. Beyond $t_{2}$, the relatively stable internal sloshing patterns are seen established although still minor irregularity being observed for the case with $75 \%$ filling ratio. The amplitude of the sloshing waves in the two liquid cabins for the lower ratio
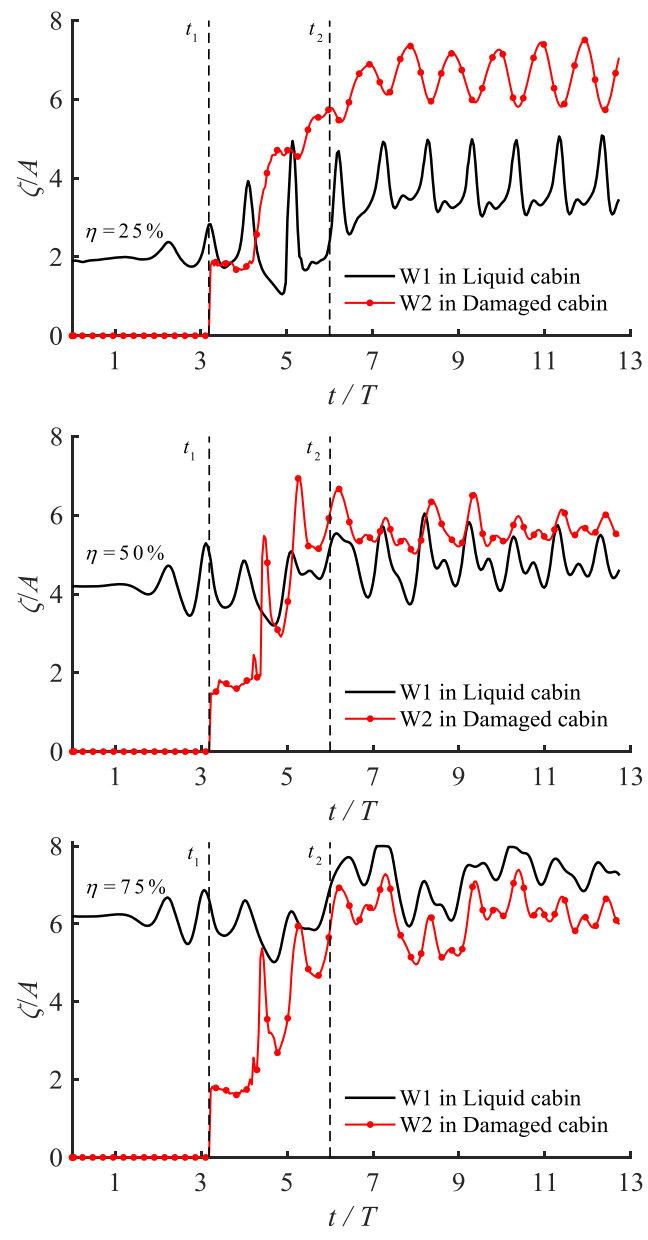

FIG. 22. Surface elevations in the damaged cabin and liquid cabin, respectively. The top: the ratio $\eta=25 \%$; the middle: the ratio $\eta=50 \%$; and the bottom: the ratio $\eta=75 \%$. 


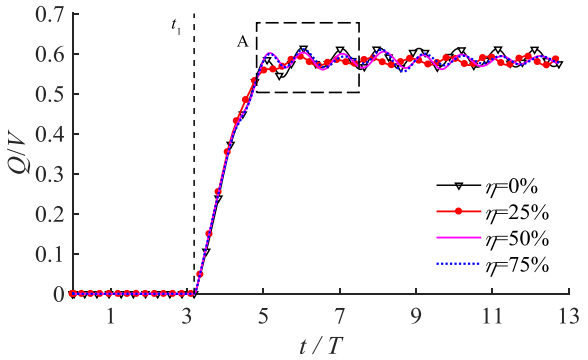

(a)

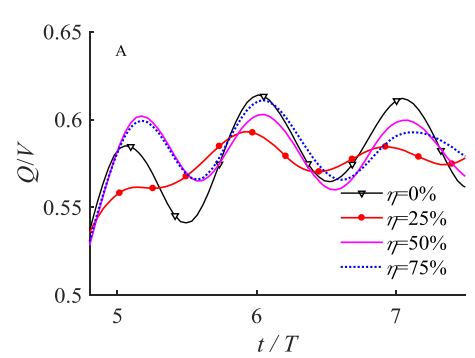

(b) of filing liquid appears to be larger. For the ratio of $\eta=25 \%$ and $\eta=50 \%$, the nonlinear sloshing waves are induced by the coupling effects with higher harmonics, doubling the frequency of the wave frequency, while a beat frequency of the difference in the frequency of the wave frequency and the sloshing frequency was detected for $\eta=75 \%$. Figure 22 shows that a clear phase difference between the sloshing waves in the damaged cabin and the liquid cabins is evident for all cases with different filling ratios. For the ratio of $\eta=25 \%$, the phase difference between the sloshing wave in the damaged cabin and the liquid cabin is approximately $\pi$, while the difference tends to decrease with approximately $\pi / 2$ for $\eta=50 \%$ and almost the same phase for $\eta=75 \%$. Based on the above results, for the smaller filled case, the external wave takes the domain effects and the nonlinear sloshing waves inside the ship increase the roll amplitude of the ship. With the increase in the filled ratio, the coupling effects by the sloshing wave in the liquid cabin and damaged cabin are enhanced.

The amount of the flooding water in the damaged ship with different liquid ratios in the two undamaged cabins is shown in Fig. 23. When the damage occurs to the middle cabin at $t_{1}$, the water floods into the cabin quickly initially showing an almost linear trend increase. This is followed by a sharp slowing down in flooding water accumulation due to a new balance of the gravity and the buoyancy of the ship being reached. Since the water exchange continues at the opening, the flooding amount fluctuates. Figure 23 shows that the amount of the flooding water fluctuates more markedly for the damaged ship with no liquid filled in the undamaged cabins. There is a clear trend that the fluctuations of flooding amount decrease with the increase in the ratio of filling liquid in the undamaged cabins due to the effect of liquid tank sloshing dampening the roll motion and consequently slowing the flow exchange at the opening.

\section{CONCLUSIONS}

An SPH model based on the strip theory is developed to study the complex coupling among flooding water, ship hull, internal liquid sloshing, and external waves. The present SPH model shows the ability to capture nonlinear phenomenon in the flooding process and to further analyze the coupling effects of various factors including the water exchange near the opening, dynamic flooding and sloshing motion, and effects on the dynamics of a damaged ship. By ignoring the flow motion along the longitudinal direction of the ship, the coupled motion in the transverse section is considered properly for a 3D ship model.
The rolling amplitude of the damaged ship decreases due to the damping effects of flooding water motion in the damaged cabin. The kinetic moment of flooding water for larger opening is dissipated faster due to the violent flooding process at the transient stage leading to the reduced amplitude of sloshing wave in the damaged cabin with the increase in opening size. The periodical inflow and outflow tend to be consistent for different opening sizes. However, the heave motion of the damaged ship in beam waves is slightly affected by the flooding process, and the damaged condition or opening is attributed to the equilibrium position of the heave motion.

When the opening occurs facing the incident wave, the flooding process is more violent at the initial flooding stage than the back one, due to the positive superposition of the moment induced by flooding water and the wave moment. The head wave causes the flooding slowing down, and the roll motion reduces more rapidly compared to the back wave case. However, the damaged ship under the back wave condition has a higher rolling frequency and larger rolling angle when the flooding reaches a dynamic equilibrium. The maximum rolling angle for both cases occurs on the opposite side of the opening.

When the damaged ship has liquid tanks, the liquid ratios have a significant effect on the new equilibrium position. The phase differences among the sloshing wave in the damaged cabin and the undamaged liquid cabins and the external wave make the coupling motion more complicated. With the increase in the liquid ratio filled in the undamaged cabins, the heeling angle decreases at the transient flooding stage leading to a reduced amplitude of the sloshing wave in the damaged cabin. There is a critical liquid ratio of approximately $50 \%$ resulting in the minimum roll motion for the simplified three cabin configuration considered in this paper.

This paper showed the great advantage of the SPH method in modeling violent free surface deformation in the flooding process. It is worth noting that the current $\mathrm{SPH}$ model requires considerable computational cost for the whole problem, especially for the external flow with small deformation. For further study, a coupled model with the SPH method for internal flow and a mesh-based method for external potential flow is currently under development for improving the efficiency of the numerical model.

\section{ACKNOWLEDGMENTS}

This paper was supported by the National Natural Science Foundation of China (Nos. 51609045 and 51879053), the Natural Science Foundation of Heilongjiang Province (No. E2017025), and the China Postdoctoral Science Foundation (No. 2016M601418). 


\section{REFERENCES}

${ }^{1}$ M. T. Tavakoli, J. Amdahl, and B. J. Leira, "Experimental investigation of oil leakage from damaged ships due to collision and grounding," Ocean Eng. 38, 1894-1907 (2011).

${ }^{2} \mathrm{P}$. Ruponen, "On the effects of non-watertight doors on progressive flooding in a damaged passenger ship,” Ocean Eng. 130, 115-125 (2017).

${ }^{3}$ F. R. Ming, A. M. Zhang, Y. Z. Xue, and S. P. Wang, "Damage characteristics of ship structures subjected to shockwaves of underwater contact explosions," Ocean Eng. 117, 359-382 (2016).

${ }^{4}$ Z. F. Zhang, L. K. Wang, and V. V. Silberschmidt, "Damage response of steel plate to underwater explosion: Effect of shaped charge liner," Int. J. Impact Eng. 103, 38-49 (2017).

${ }^{5} \mathrm{Z}$. Gao and D. Vassalos, "The dynamics of the floodwater and the damaged ship in waves," J. Hydrodyn. 27, 689-695 (2015).

${ }^{6}$ E. Begovic, G. Mortola, A. Incecik, and A. Day, "Experimental assessment of intact and damaged ship motions in head, beam and quartering seas," Ocean Eng. 72, 209-226 (2013).

${ }^{7}$ T. A. Santos and C. G. Soares, "Numerical assessment of factors affecting the survivability of damaged ro-ro ships in waves," Ocean Eng. 36, 797-809 (2009).

${ }^{8}$ C. Khaddaj-Mallat, J. M. Rousset, and P. Ferrant, "The transient and progressive flooding stages of damaged ro-ro vessels: A systematic review of entailed factors," J. Offshore Mech. Arct. Eng. 133, 031105 (2011).

${ }^{9}$ J. De Kat and R. Van't Veer, "Mechanisms and physics leading to the capsize of damaged ships," in Proceedings of the Fifth International Workshop on Ship Stability, Trieste, Italy, 2001.

${ }^{10}$ Y. Ikeda, S. Shimoda, and Y. Takeuchi, "Experimental studies on transient motion and time to sink of a damaged large passenger ship," in Proceedings of the Eight International Conference on the Stability of Ships and Ocean Vehicles (STAB 2003), Madrid, Spain, 2003.

${ }^{11}$ T. Manderbacka, P. Ruponen, J. Kulovesi, and J. Matusiak, "Model experiments of the transient response to flooding of the box shaped barge," J. Fluids Struct. 57, 127-143 (2015).

${ }^{12}$ L. Letizia, D. Vassalos, and A. Jasionowski, "New insights into ship-floodwatersea dynamics," Int. Shipbuild. Prog. 51, 273-291 (2004).

${ }^{13}$ D. Lee, S. Y. Hong, and G. J. Lee, "Theoretical and experimental study on dynamic behavior of a damaged ship in waves," Ocean Eng. 34, 21-31 (2007).

${ }^{14}$ D. Spanos and A. Papanikolaou, "Numerical study of the damage stability of ships in intermediate stages of flooding," in Proceedings of the Fifth International Workshop on Ship Stability, Trieste, Italy, 2001.

${ }^{15} \mathrm{Y}$. Ikeda and Y. Ma, "An experimental study on large roll motion in intermediate stage of flooding due to sudden ingress of water," in Proceedings of the Seventh International Conference on the Stability of Ships and Ocean Vehicles (STAB 2000), Launceston, Australia, 2000.

${ }^{16}$ T. Manderbacka, T. Mikkola, P. Ruponen, and J. Matusiak, "Transient response of a ship to an abrupt flooding accounting for the momentum flux," J. Fluids Struct. 57, 108-126 (2015).

${ }^{17}$ T. A. Santos and C. Guedes Soares, "Study of damaged ship motions taking into account floodwater dynamics," J. Mar. Sci. Technol. 13, 291-307 (2008).

${ }^{18}$ H.-S. Chan, M. Atlar, and A. Incecik, "Large-amplitude motion responses of a ro-ro ship to regular oblique waves in intact and damaged conditions," J. Mar. Sci. Technol. 7, 91-99 (2002).

${ }^{19} \mathrm{E}$. Korkut, M. Atlar, and A. Incecik, "An experimental study of motion behaviour with an intact and damaged ro-ro ship model," Ocean Eng. 31, 483-512 (2004).

${ }^{20}$ A. Papanikolaou, "Review of damage stability of ships-recent developments and trends," in Proceedings 10th Int. Symposium on Practical Design of Ships and Other Floating Structures, Houston, 2007.

${ }^{21}$ C. Khaddaj-Mallat, B. Alessandrini, J.-M. Rousset, and P. Ferrant, "An experimental study on the flooding of a damaged passenger ship," Ships Offshore Struct. 7, 55-71 (2012).

${ }^{22}$ F. R. Ming, A. M. Zhang, H. Cheng, and P. N. Sun, "Numerical simulation of a damaged ship cabin flooding in transversal waves with smoothed particle hydrodynamics method," Ocean Eng. 165, 336-352 (2018).
${ }^{23}$ W. Zhao, J. Yang, Z. Hu, and L. Tao, "Coupled analysis of nonlinear sloshing and ship motions," Appl. Ocean Res. 47, 85-97 (2014).

${ }^{24}$ D. Zhao, Z. Hu, G. Chen, X. Chen, and X. Feng, "Coupling analysis between vessel motion and internal nonlinear sloshing for FLNG applications," J. Fluids Struct. 76, 431-453 (2018).

${ }^{25}$ L. Palazzi and J. De Kat, "Model experiments and simulations of a damaged ship with air flow taken into account," Mar. Technol. 41, 38-44 (2004).

${ }^{26}$ P. Ruponen, P. Kurvinen, I. Saisto, and J. Harras, "Air compression in a flooded tank of a damaged ship," Ocean Eng. 57, 64-71 (2013).

${ }^{27}$ X. Y. Cao, F. R. Ming, A. M. Zhang, and L. Tao, "Multi-phase SPH modelling of air effect on the dynamic flooding of a damaged cabin," Comput. Fluids 163, 7-19 (2018).

${ }^{28}$ Z. Gao, Y. Wang, Y. Su, and L. Chen, "Numerical study of damaged ship's compartment sinking with air compression effect," Ocean Eng. 147, 68-76 (2018).

${ }^{29}$ L. Shen and D. Vassalos, Applications of 3D Parallel SPH for Sloshing and Flooding (Springer, 2011), pp. 709-721.

${ }^{30} \mathrm{M}$. Acanfora and A. Cirillo, "On the development of a fast modeling of floodwater effects on ship motions in waves," Proc. Inst. Mech. Eng., Part M 231, 877-887 (2017).

${ }^{31}$ A. Zhang, P. Sun, and F. Ming, "An SPH modeling of bubble rising and coalescing in three dimensions," Comput. Methods Appl. Mech. Eng. 294, 189-209 (2015).

${ }^{32} \mathrm{X}$. Xu, "An improved SPH approach for simulating 3D dam-break flows with breaking waves," Comput. Methods Appl. Mech. Eng. 311, 723-742 (2016).

${ }^{33}$ J. Cercos-Pita, M. Antuono, A. Colagrossi, and A. Souto-Iglesias, "SPH energy conservation for fluid-solid interactions," Comput. Methods Appl. Mech. Eng. 317, 771-791 (2017).

${ }^{34} \mathrm{M}$. Shadloo, G. Oger, and D. Le Touzé, "Smoothed particle hydrodynamics method for fluid flows, towards industrial applications: Motivations, current state, and challenges," Comput. Fluids 136, 11-34 (2016).

${ }^{35}$ B. Bouscasse, M. Antuono, A. Colagrossi, and C. Lugni, "Numerical and experimental investigation of nonlinear shallow water sloshing," Int. J. Nonlinear Sci. Numer. Simul. 14, 123-138 (2013).

${ }^{36} \mathrm{P}$. Caron, M. Cruchaga, and A. Larreteguy, "Study of 3D sloshing in a vertical cylindrical tank," Phys. Fluids 30, 082112 (2018).

${ }^{37}$ S. Marrone, M. Antuono, A. Colagrossi, G. Colicchio, D. Le Touzé, and G. Graziani, " $\delta$-SPH model for simulating violent impact flows ," Comput. Methods Appl. Mech. Eng. 200, 1526-1542 (2011).

${ }^{38}$ K. Gong, S. Shao, H. Liu, B. Wang, and S.-K. Tan, "Two-phase SPH simulation of fluid-structure interactions," J. Fluids Struct. 65, 155-179 (2016).

${ }^{39}$ B. Bouscasse, A. Colagrossi, S. Marrone, and A. Souto-Iglesias, "SPH modelling of viscous flow past a circular cylinder interacting with a free surface," Comput. Fluids 146, 190-212 (2017).

${ }^{40}$ S. Prasanna Kumar, B. Patnaik, and K. Ramamurthi, "Prediction of air blast mitigation in an array of rigid obstacles using smoothed particle hydrodynamics," Phys. Fluids 30, 046105 (2018).

${ }^{41}$ X. Cao, F. Ming, and A. Zhang, "Sloshing in a rectangular tank based on SPH simulation," Appl. Ocean Res. 47, 241-254 (2014).

${ }^{42}$ B. Bouscasse, A. Colagrossi, A. Souto-Iglesias, and J. Cercos-Pita, "Mechanical energy dissipation induced by sloshing and wave breaking in a fully coupled angular motion system. I. Theoretical formulation and numerical investigation," Phys. Fluids 26, 033103 (2014).

${ }^{43}$ B. Bouscasse, A. Colagrossi, A. Souto-Iglesias, and J. Cercos-Pita, "Mechanical energy dissipation induced by sloshing and wave breaking in a fully coupled angular motion system. II. Experimental investigation," Phys. Fluids 26, 033104 (2014).

${ }^{44}$ B. Bouscasse, A. Colagrossi, S. Marrone, and M. Antuono, "Nonlinear water wave interaction with floating bodies in SPH," J. Fluids Struct. 42, 112-129 (2013). ${ }^{45}$ H. Cheng, A. M. Zhang, and F. R. Ming, "Study on coupled dynamics of ship and flooding water based on experimental and SPH methods," Phys. Fluids 29, 107101 (2017).

${ }^{46}$ R. Vacondio, B. Rogers, P. Stansby, and P. Mignosa, "Shallow water SPH for flooding with dynamic particle coalescing and splitting," Adv. Water Resour. 58, 10-23 (2013). 
${ }^{47}$ D. Le Touzé, A. Marsh, G. Oger, P. Guilcher, C. Khaddaj-Mallat, B. Alessandrini, and P. Ferrant, "SPH simulation of green water and ship flooding scenarios," J. Hydrodyn. 22, 231-236 (2010).

${ }^{48} \mathrm{~J}$. P. Berenger, "A perfectly matched layer for the absorption of electromagnetic waves," J. Comput. Phys. 114, 185-200 (1994).

${ }^{49}$ D. Molteni, R. Grammauta, and E. Vitanza, "Simple absorbing layer conditions for shallow wave simulations with smoothed particle hydrodynamics," Ocean Eng. 62, 78-90 (2013).

${ }^{50}$ J. J. Monaghan, "Smoothed particle hydrodynamics," Annu. Rev. Astron. Astrophys. 30, 543-574 (1992).

${ }^{51}$ A. Colagrossi and M. Landrini, "Numerical simulation of interfacial flows by smoothed particle hydrodynamics," J. Comput. Phys. 191, 448-475 (2003).

${ }^{52}$ G. R. Liu and M. B. Liu, Smoothed Particle Hydrodynamics: A Meshfree Particle Method (World Scientific, 2003), pp. 35-47.

${ }^{53} \mathrm{~J}$. J. Monaghan and R. A. Gingold, "Shock simulation by the particle method SPH,” J. Comput. Phys. 52, 374-389 (1983).

${ }^{54} \mathrm{~J}$. J. Monaghan, "Simulating free surface flows with SPH," J. Comput. Phys. 110, 399-406 (1994).

${ }^{55}$ D. Meringolo, A. Colagrossi, S. Marrone, and F. Aristodemo, "On the filtering of acoustic components in weakly-compressible SPH simulations," J. Fluids Struct. 70, 1-23 (2017).

${ }^{56}$ S. Marrone, Ph.D. thesis, Universita di Roma Sapienza, 2012.

${ }^{57}$ S. Marrone, A. Colagrossi, A. Di Mascio, and D. Le Touzé, "Prediction of energy losses in water impacts using incompressible and weakly compressible models," J. Fluids Struct. 54, 802-822 (2015).

${ }^{58}$ A. Colagrossi, M. Antuono, and D. Le Touzé, "Theoretical considerations on the free-surface role in the smoothed-particle-hydrodynamics model," Phys. Rev. E 79, 056701 (2009).

${ }^{59}$ A. M. Zhang, P. N. Sun, F. R. Ming, and A. Colagrossi, "Smoothed particle hydrodynamics and its applications in fluid-structure interactions," J. Hydrodyn. 29, 187-216 (2017).

${ }^{60} \mathrm{~J}$. Bonet and T. S. Lok, "Variational and momentum preservation aspects of smooth particle hydrodynamic formulations," Comput. Methods Appl. Mech. Eng. 180, 97-115 (1999).

${ }^{61}$ F. Macia Lang, A. Souto Iglesias, M. Antuono, and A. Colagrossi, "Benefits of using a Wendland kernel for free-surface flows," in Proceedings of 6th ERCOFTAC SPHERIC Workshop on SPH Applications, Hamburgo, Alemania, 2011.
${ }^{62}$ N. Grenier, M. Antuono, A. Colagrossi, D. Le Touzé, and B. Alessandrini, "An Hamiltonian interface SPH formulation for multi-fluid and free surface flows," J. Comput. Phys. 228, 8380-8393 (2009).

${ }^{63}$ A. M. Zhang, X. Y. Cao, F. R. Ming, and Z. F. Zhang, "Investigation on a damaged ship model sinking into water based on three dimensional SPH method," Appl. Ocean Res. 42, 24-31 (2013).

${ }^{64}$ P. Sun, A. Colagrossi, S. Marrone, and A. Zhang, "The $\delta$ plus-SPH model: Simple procedures for a further improvement of the SPH scheme," Comput. Methods Appl. Mech. Eng. 315, 25-49 (2017).

${ }^{65} \mathrm{M}$. Antuono, S. Marrone, A. Colagrossi, and B. Bouscasse, "Energy balance in the $\delta$-SPH scheme," Comput. Methods Appl. Mech. Eng. 289, 209-226 (2015).

${ }^{66}$ P.-N. Sun, A. Colagrossi, and A.-M. Zhang, "Numerical simulation of the selfpropulsive motion of a fishlike swimming foil using the $\delta+-\mathrm{SPH}$ model," Theor, Appl. Mech. Lett. 8, 115-125 (2018).

${ }^{67}$ M. D. Green and J. Peiró, "Long duration SPH simulations of sloshing in tanks with a low fill ratio and high stretching," Comput. Fluids 174, 179-199 (2018).

${ }^{68}$ B. Korvin-Kroukovsky, "Investigation of ship motions in regular waves," Trans. SNAME 63, 386-435 (1955).

${ }^{69}$ S. Adami, X. Y. Hu, and N. A. Adams, "A generalized wall boundary condition for smoothed particle hydrodynamics," J. Comput. Phys. 231, 7057-7075 (2012).

${ }^{70}$ P. N. Sun, F. R. Ming, and A. M. Zhang, "Numerical simulation of interactions between free surface and rigid body using a robust SPH method," Ocean Eng. 98, 32-49 (2015).

${ }^{71}$ R. G. Dean and R. A. Dalrymple, Water Wave Mechanics for Engineers and Scientists (World Scientific Publishing Company, 1991).

${ }^{72}$ P. Wang, A.-M. Zhang, F. Ming, P. Sun, and H. Cheng, "A novel nonreflecting boundary condition for fluid dynamics solved by smoothed particle hydrodynamics," J. Fluid Mech. 860, 81-114 (2019).

${ }^{73}$ A. Modave, É. Deleersnijder, and É. J. Delhez, "On the parameters of absorbing layers for shallow water models,” Ocean Dyn. 60, 65-79 (2010).

${ }^{74}$ I. Hadžić, J. Hennig, M. Perić, and Y. Xing-Kaeding, "Computation of flow-induced motion of floating bodies," Appl. Math. Modell. 29, 1196-1210 (2005).

${ }^{75}$ F. Abaqus, "ABAQUS analysis user's manual," Dassault Systemes, VélizyVillacoublay, France, 2009. 